\title{
Calix[4]arene Derivatives Monosubstituted at all Four Methylene Bridges
}

\author{
Ishay Columbus and Silvio E. Biali \\ Department of Organic Chemistry, The Hebrew University of Jerusalem \\ Jerusalem 91904, Israel
}

Supporting Information

\author{
Page S2: Figure S1. ${ }^{1} \mathrm{H}$ NMR spectrum of 2a in $\mathrm{CDCl}_{3}$ \\ Page S3: Figure S2. ${ }^{1} \mathrm{H}$ NMR spectrum of 4 in $\mathrm{CDCl}_{3}$. \\ Page S4: Figure S3. ${ }^{13} \mathrm{C}$ NMR spectrum of 4 in $\mathrm{CDCl}_{3}$. \\ Page S5: Figure S4. ${ }^{1} \mathrm{H}$ NMR spectrum of $\mathbf{6 a}$ in $\mathrm{CDCl}_{3}$. \\ Page S6: Figure S5. ${ }^{1} \mathrm{H}$ NMR spectrum of 6 a in $\mathrm{CDCl}_{3}$ (expansion) \\ Page S7: Figure S6. ${ }^{1} \mathrm{H}$ NMR spectrum of $\mathbf{6 c}$ in $\mathrm{CDCl}_{3}$. \\ Page S8: Figure S7. ${ }^{13} \mathrm{C}$ NMR spectrum of $\mathbf{6 c}$ in $\mathrm{CDCl}_{3}$. \\ Page S9: Figure S8. ${ }^{1} \mathrm{H}$ NMR spectrum of $\mathbf{6 d}$ in $\mathrm{CDCl}_{3}$. \\ Page S10: Figure S9. ${ }^{13} \mathrm{C}$ NMR spectrum of $6 \mathbf{d}$ in $\mathrm{CDCl}_{3}$. \\ Page S11: Figure S10. ${ }^{1} \mathrm{H}$ NMR spectrum of $\mathbf{6} \mathbf{f}$ in $\mathrm{CDCl}_{3}$. \\ Page S12: Figure S11. ${ }^{13} \mathrm{C}$ NMR spectrum of 6 f in $\mathrm{CDCl}_{3}$. \\ Page S13: Figure S12. ${ }^{1} \mathrm{H}$ NMR spectrum of 8 in $\mathrm{CDCl}_{3}$. \\ Page S14: Figure S13. ${ }^{13} \mathrm{C}$ NMR spectrum of 8 in $\mathrm{CDCl}_{3}$. \\ Page S15: Figure S14. ${ }^{1} \mathrm{H}$ NMR spectrum of 9 in acetone- $\mathrm{d}_{6}$. \\ Page S16: Figure S15. ${ }^{13} \mathrm{C}$ NMR spectrum of 9 in acetone- $\mathrm{d}_{6}$. \\ Page S17: Figure S16. ${ }^{1} \mathrm{H}$ NMR spectrum of 10 in $\mathrm{CDCl}_{3}$. \\ Page S18: Figure S17. ${ }^{13} \mathrm{C}$ NMR spectrum of $\mathbf{1 0}$ in $\mathrm{CDCl}_{3}$. \\ Page S19: Figure S18. ${ }^{1} \mathrm{H}$ NMR spectrum of 11 in $\mathrm{CDCl}_{3}$ \\ Page S20: Figure S19. ${ }^{13} \mathrm{C}$ NMR spectrum of 11 in $\mathrm{CDCl}_{3}$. \\ Page S21: Figure S20. ${ }^{1} \mathrm{H}$ NMR spectrum of 12 in $\mathrm{CDCl}_{3}$ \\ Page S22: Figure S21. ${ }^{13} \mathrm{C}$ NMR spectrum of 12 in $\mathrm{CDCl}_{3}$. \\ Page S23: Figure S22. ${ }^{1} \mathrm{H}$ NMR spectrum of 14 in $\mathrm{CDCl}_{3}$ \\ Page S24: Figure S23. ${ }^{13} \mathrm{C}$ NMR spectrum of 14 in $\mathrm{CDCl}_{3}$. \\ Page S25: Final minimized coordinates (MM3) of the $r c c c$ isomer of $\mathbf{1 5}$, cone conformation \\ (tetraequatorial). \\ Page S27: Final minimized coordinates (MM3) of the $r c c c$ isomer of 15, partial cone \\ conformation (diequatorial-diisoclinal (I)). \\ Page S29: Final minimized coordinates (MM3) of the rcct isomer of 15, cone conformation \\ (triequatorial-axial). \\ Page S31: Final minimized coordinates (MM3) of the rcct isomer of 15, partial cone \\ conformation (diequatorial-isoclinal (I)-isoclinal(II)). \\ Page S33: Final minimized coordinates (MM3) of the $r c t t$ isomer of 15, cone conformation \\ (diequatorial-diaxial). \\ Page S35: Final minimized coordinates (MM3) of the $r c t t$ isomer of 15, partial cone \\ conformation (diequatorial-diisoclinal (II)). \\ Page S37: Final minimized coordinates (MM3) of the $r c t t$ isomer of 15, partial cone \\ conformation (diequatorial-diisoclinal (II)).
}


Figure S1. ${ }^{1} \mathrm{H}$ NMR spectrum of $\mathbf{2 a}$ in $\mathrm{CDCl}_{3}$
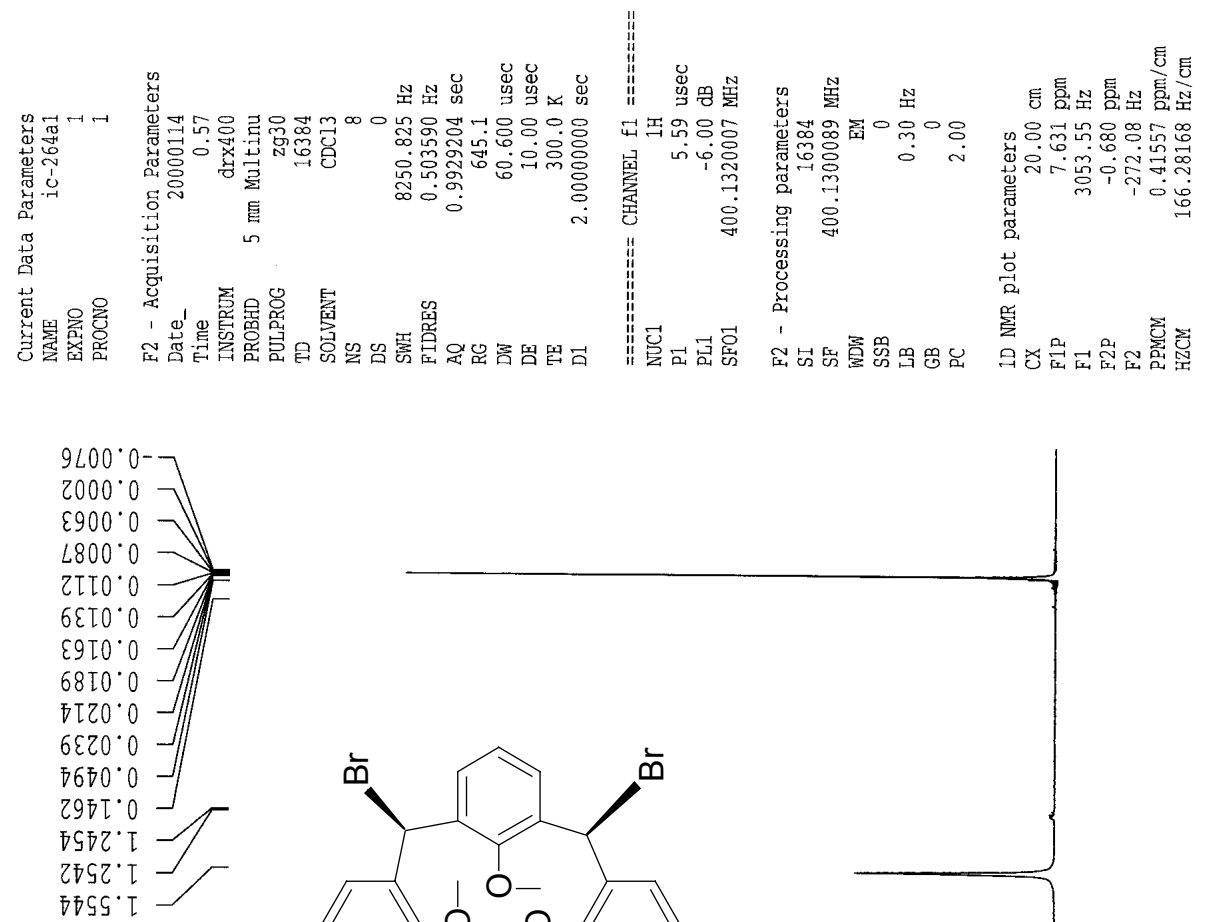

tags. I
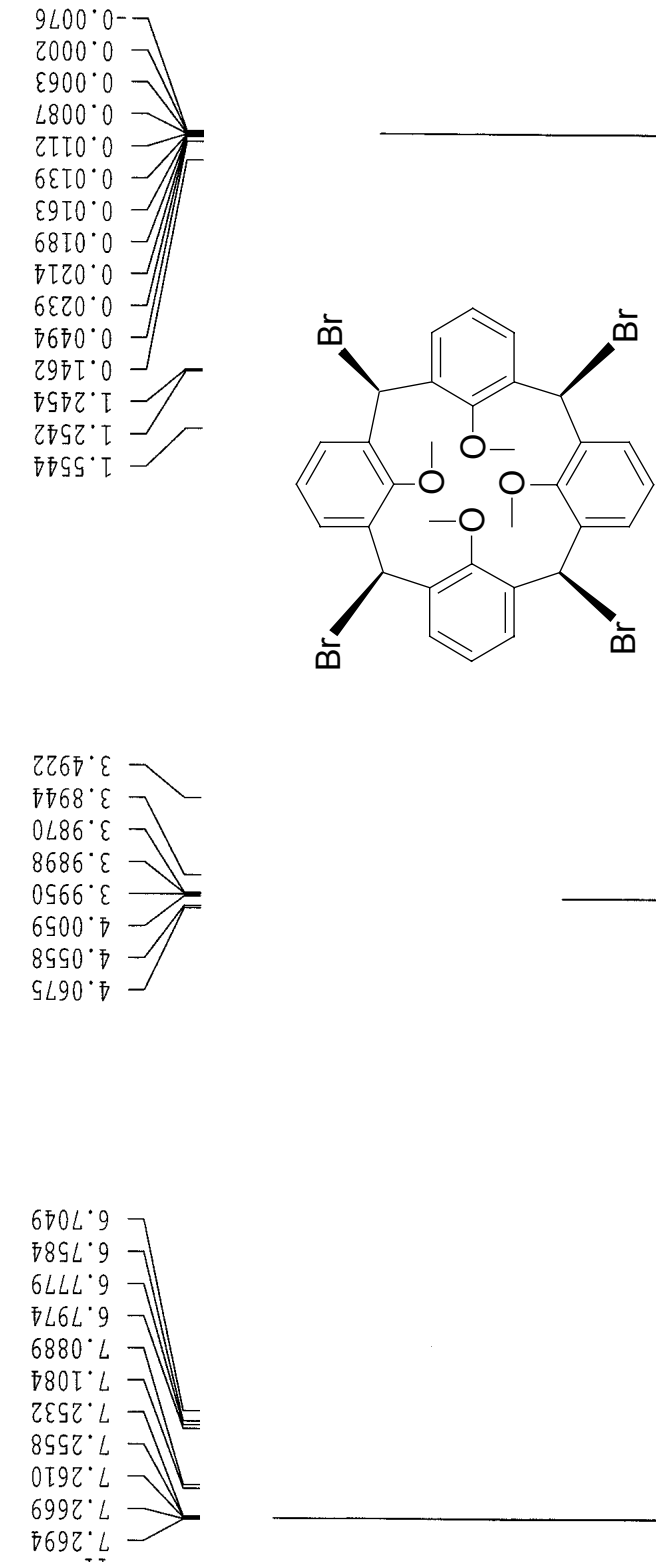

ưdd

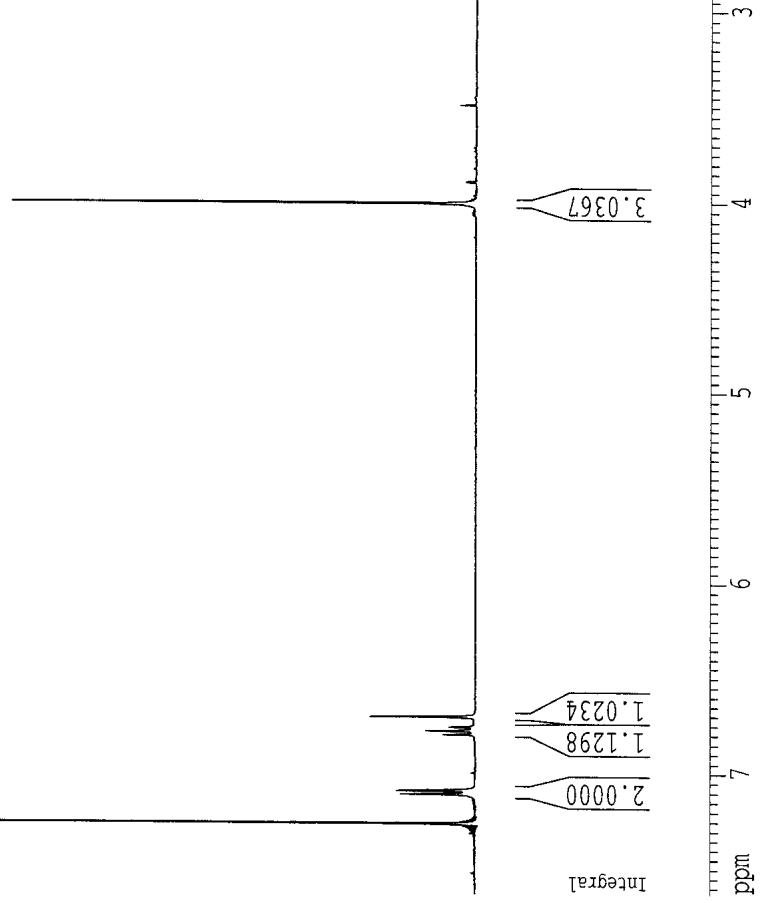


Figure S2. ${ }^{1} \mathrm{H}$ NMR spectrum of 4 in $\mathrm{CDCl}_{3}$.
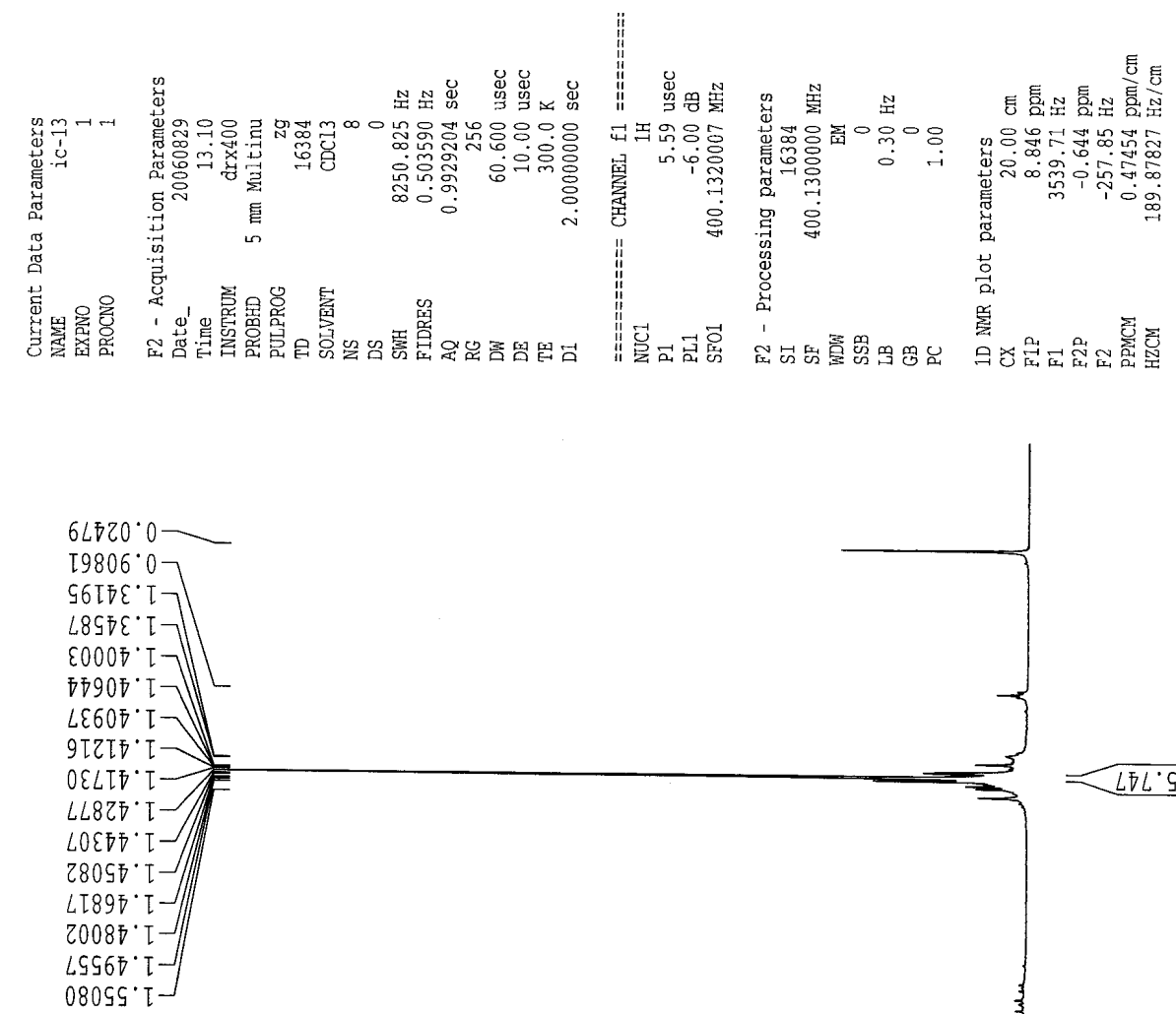

$0 z \S \varsigma \varepsilon^{\cdot} \varepsilon$

T9IIE'9-

$80582^{\circ} L-$

$\left\lceil 5068^{\circ}\right.$

โ2968. L

STLZZ:8

DLZEZ:8

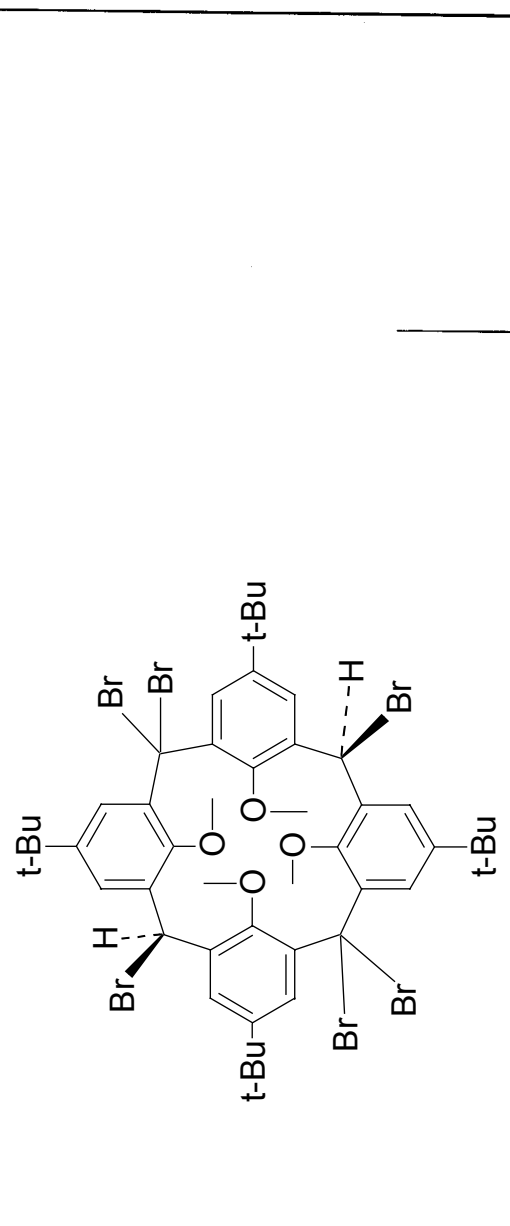

$\angle T L \cdot \underline{\underline{\varepsilon}}$

urdd

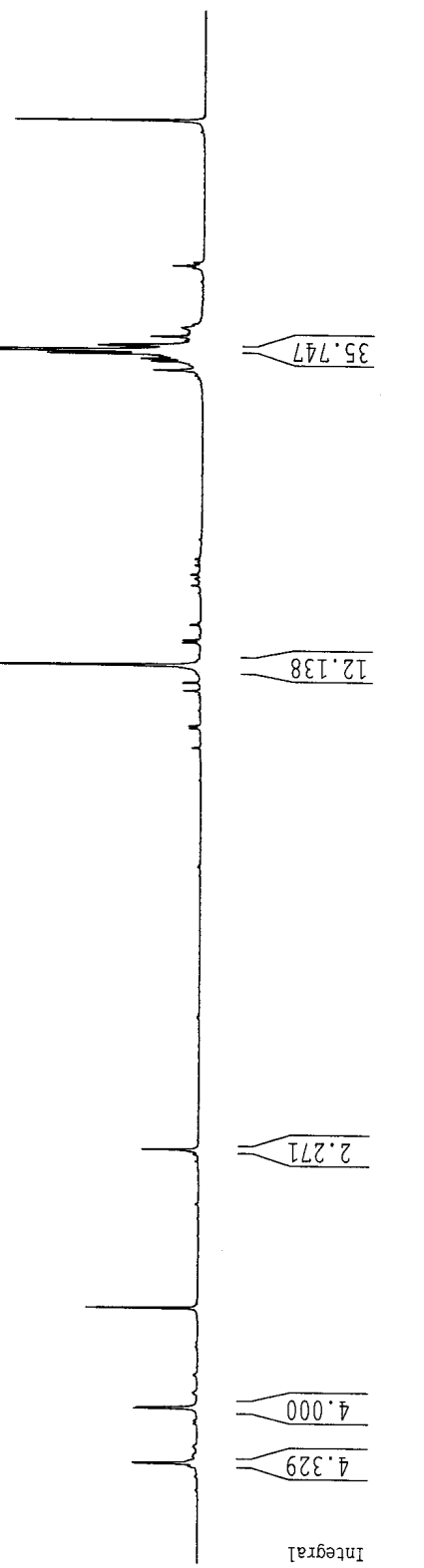


Figure S3. ${ }^{13} \mathrm{C}$ NMR spectrum of $\mathbf{4}$ in $\mathrm{CDCl}_{3}$.
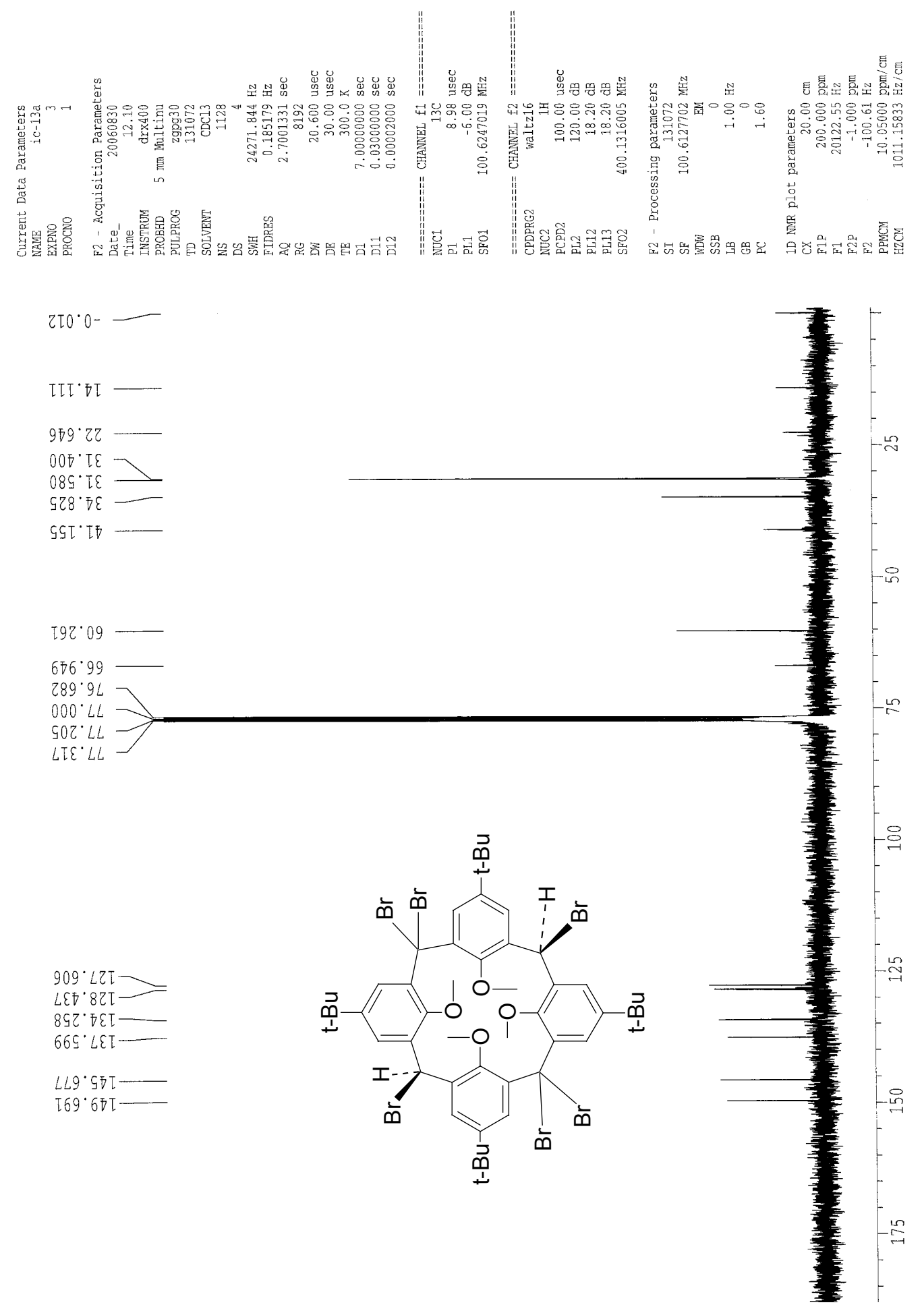
Figure S4. ${ }^{1} \mathrm{H}$ NMR spectrum of $\mathbf{6 a}$ in $\mathrm{CDCl}_{3}$.
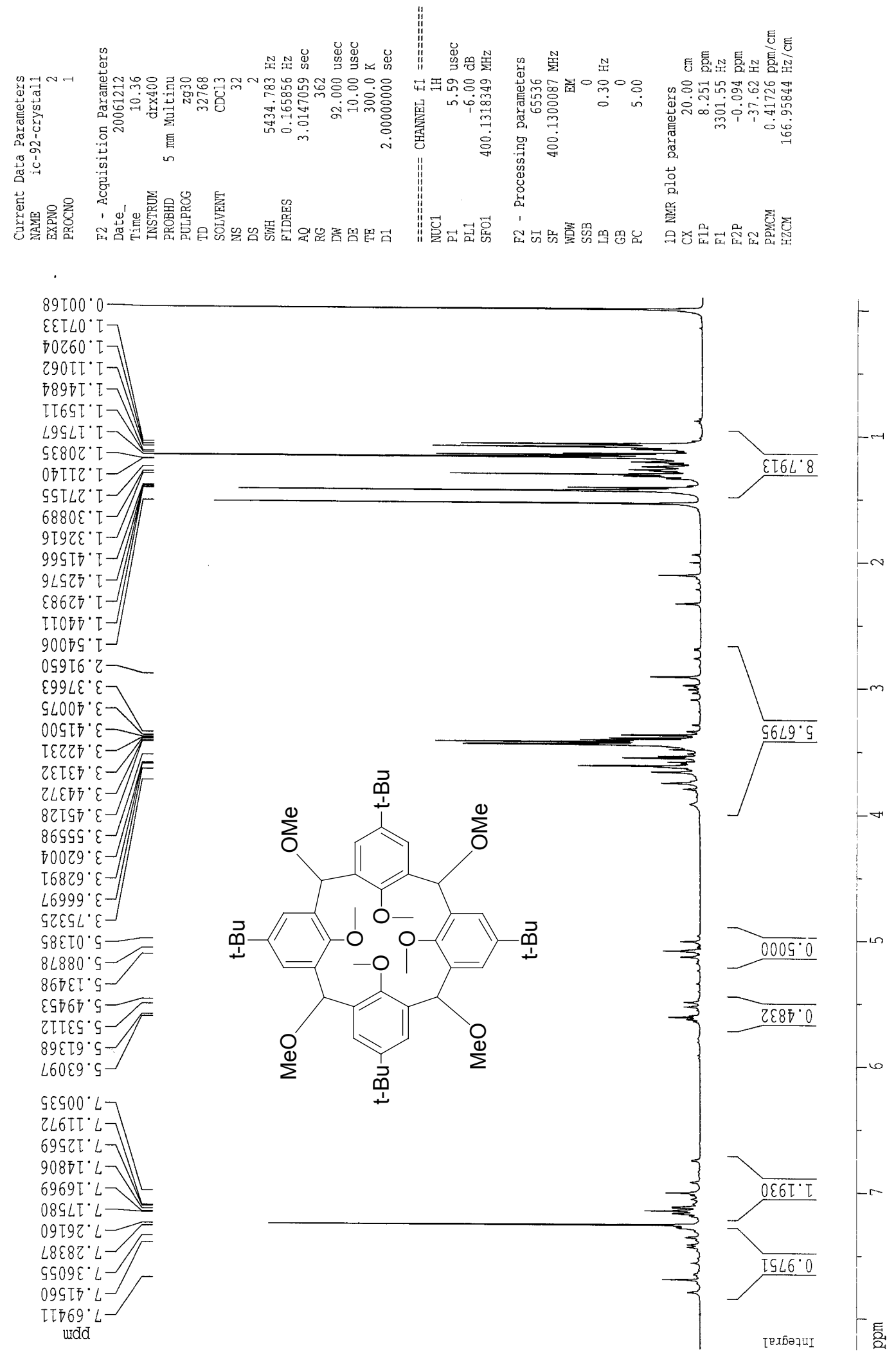
Figure S5. ${ }^{1} \mathrm{H}$ NMR spectrum of $\mathbf{6 a}$ in $\mathrm{CDCl}_{3}$ (expansion)
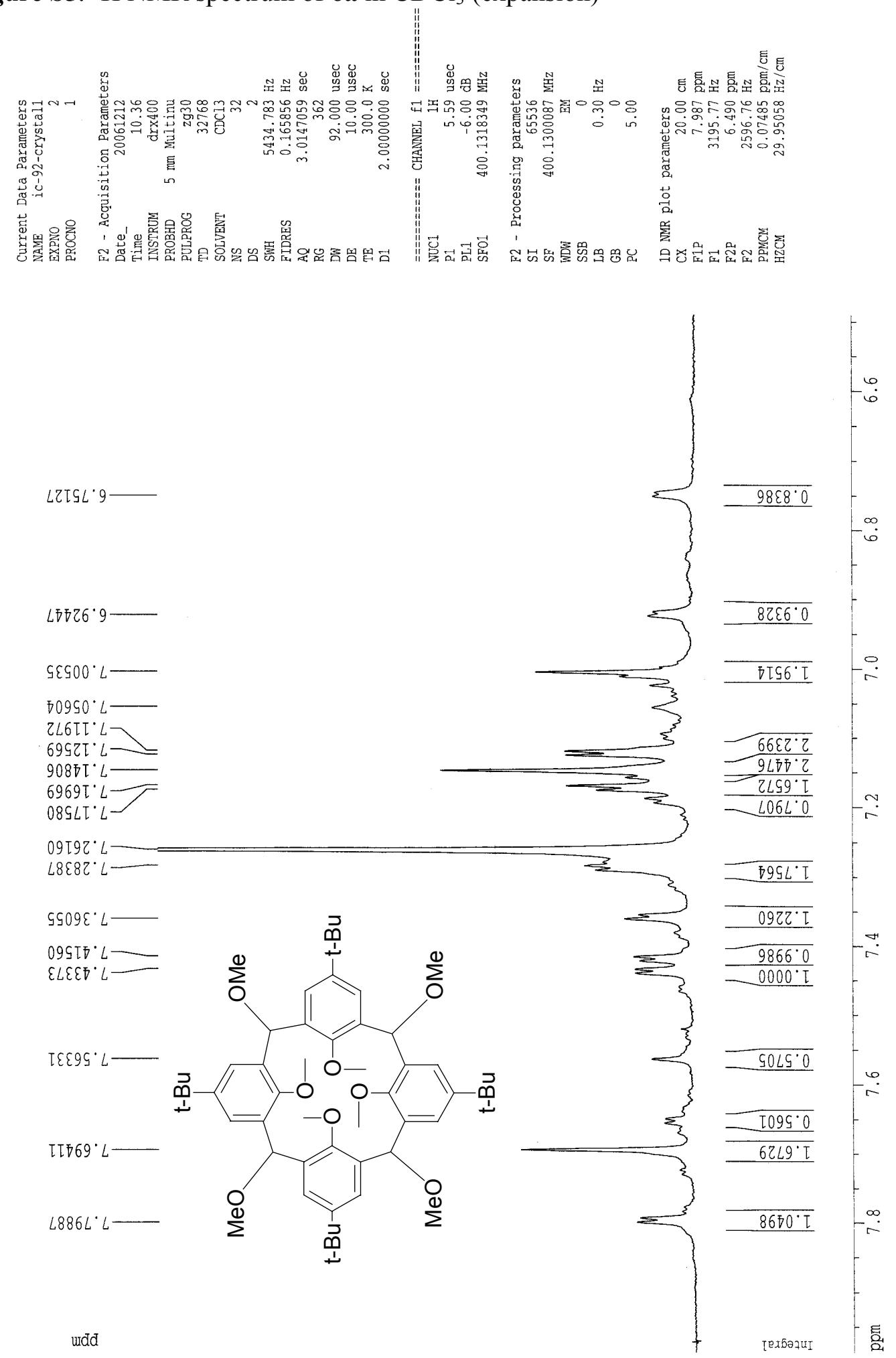
Figure S6. ${ }^{1} \mathrm{H} \mathrm{NMR}$ spectrum of $\mathbf{6 c}$ in $\mathrm{CDCl}_{3}$.
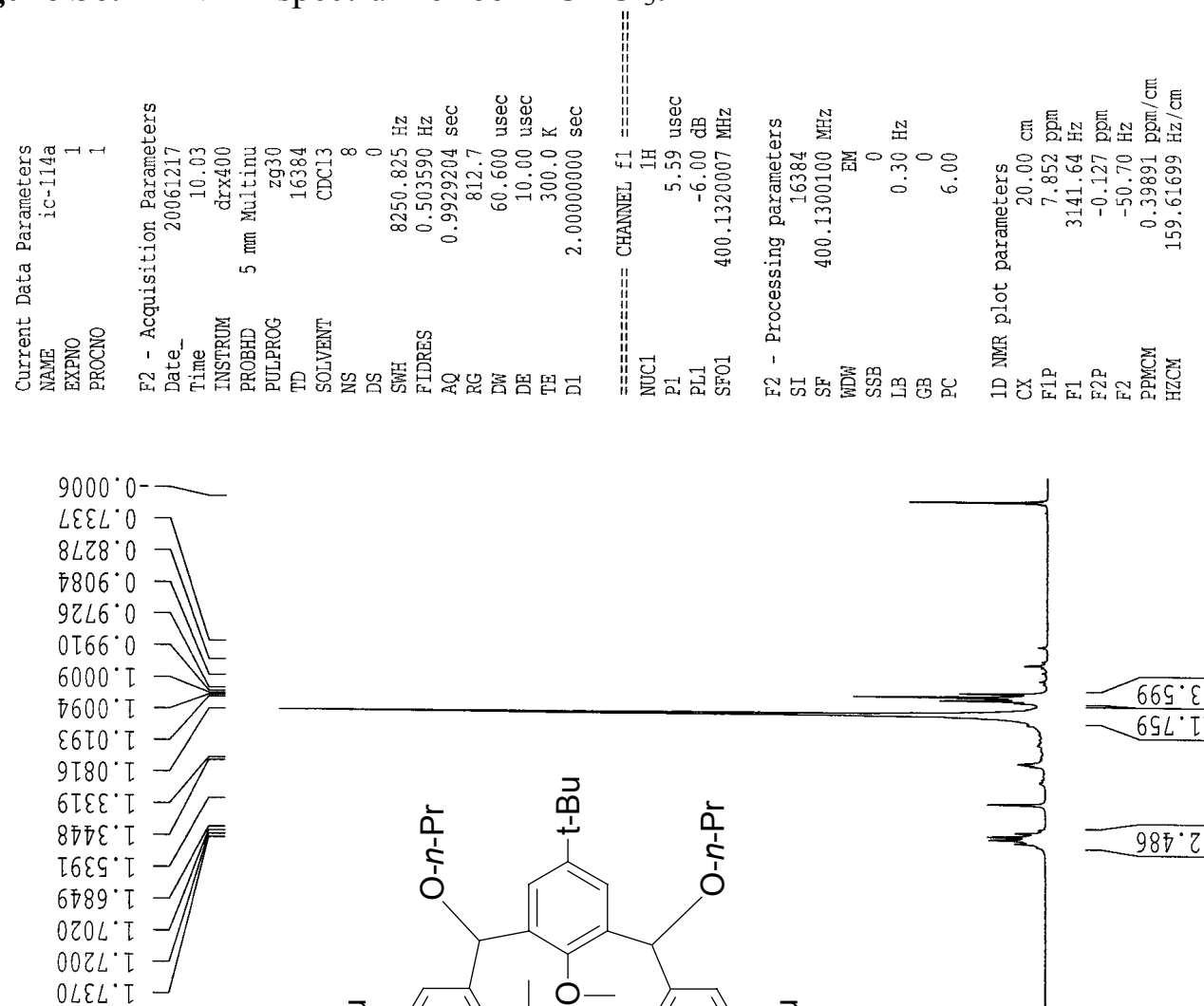

हITS. $9 \angle Z S^{\circ} \varepsilon$ $8 \varepsilon 7 S^{\circ} \varepsilon$

$6 \angle 68^{\circ} \varepsilon$

$\varepsilon L \varepsilon \sigma^{\circ} \varepsilon \longrightarrow$ $\varsigma 696^{\circ} \varepsilon$

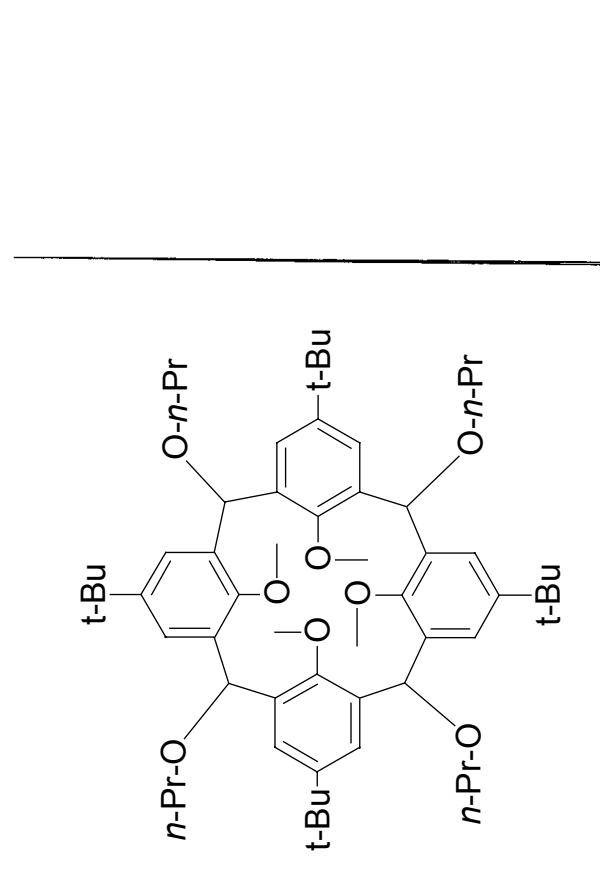

$72 L 8 \cdot 9$

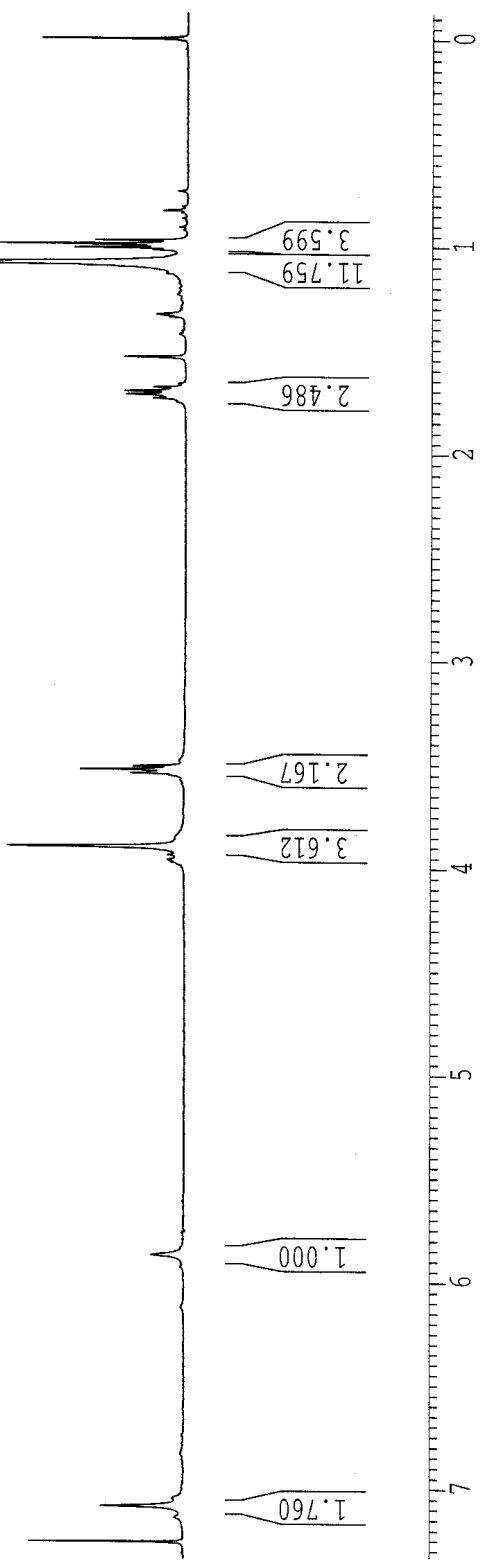


Figure S7. ${ }^{13} \mathrm{C}$ NMR spectrum of $6 \mathbf{c}$ in $\mathrm{CDCl}_{3}$.

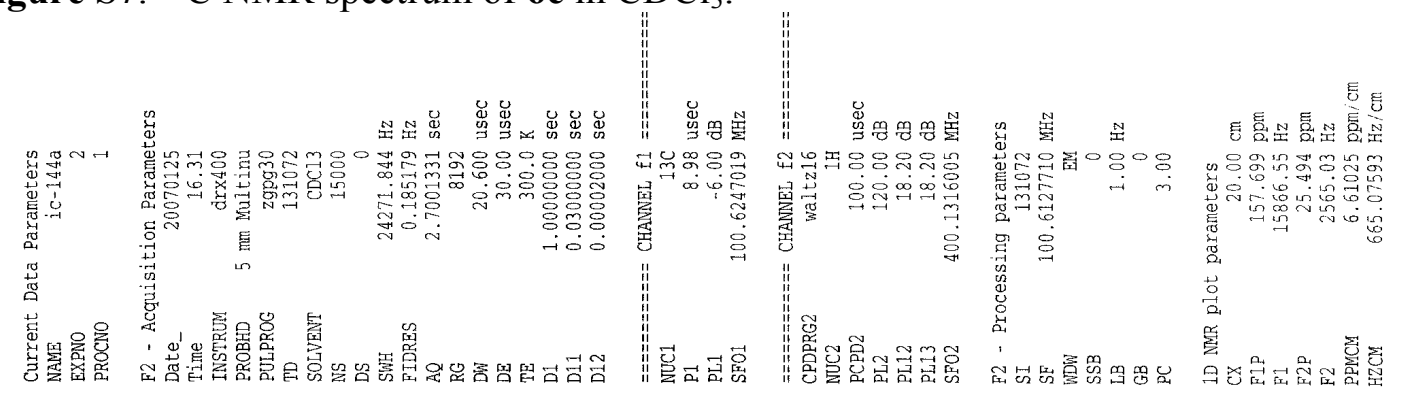

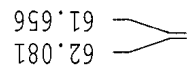

$\varepsilon \subseteq L \cdot 0 L$

T89.9L

$666^{\circ} 9 \mathrm{~L}$

9TE'LL

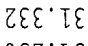
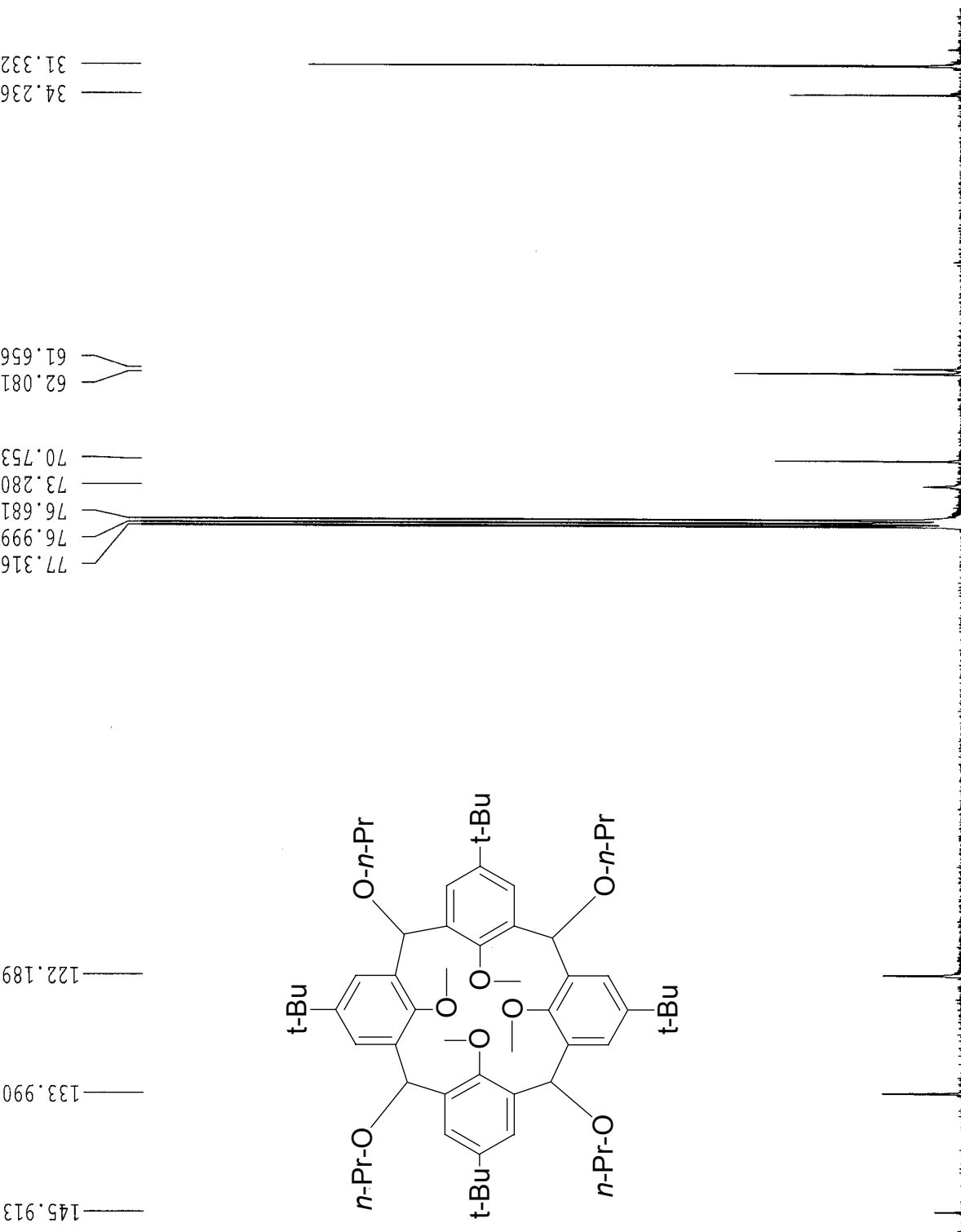

$96 T^{\prime} \varepsilon 5 T-25$
urdd

$-\infty$
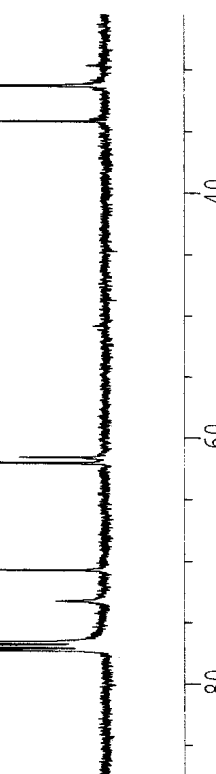
Figure S8. ${ }^{1} \mathrm{H}$ NMR spectrum of $\mathbf{6 d}$ in $\mathrm{CDCl}_{3}$.

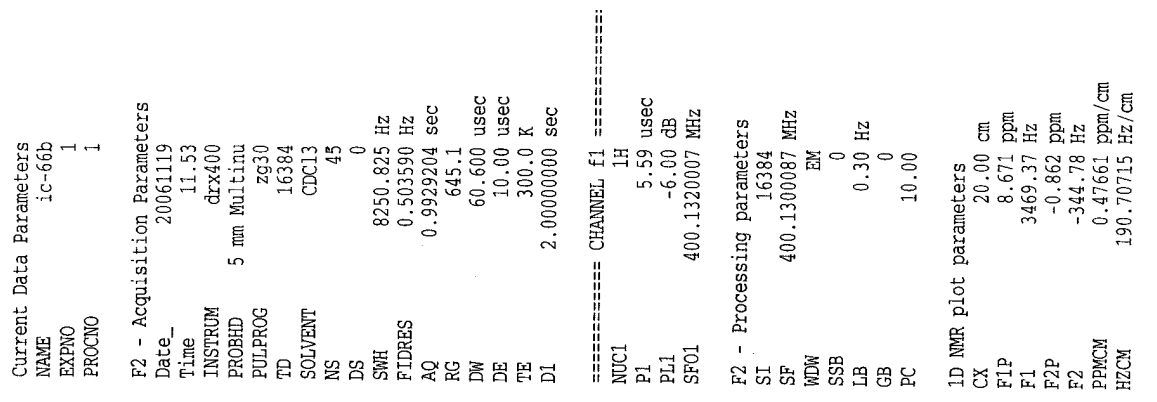

₹9LLL' $\varepsilon-$
I826L' $\varepsilon-1$

I826 L $L^{\circ} \varepsilon$

$\angle 9 L T 6 \cdot \varepsilon-$

$\varepsilon 8960.9-$

$20680^{\circ} \mathrm{L}-$ $\varepsilon \mp T 9 Z^{\circ} L$

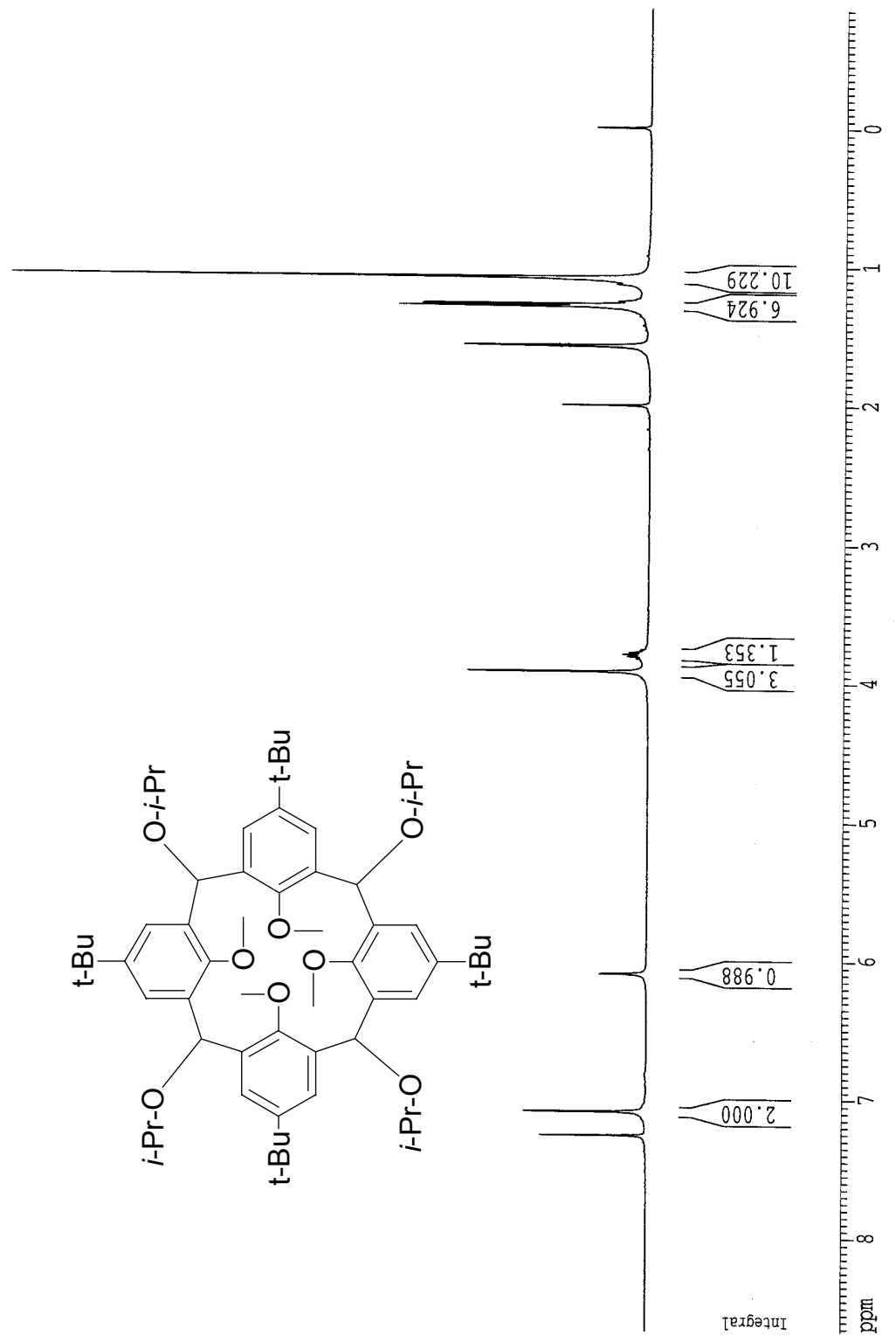


Figure S9. ${ }^{13} \mathrm{C}$ NMR spectrum of $\mathbf{6 d}$ in $\mathrm{CDCl}_{3}$.

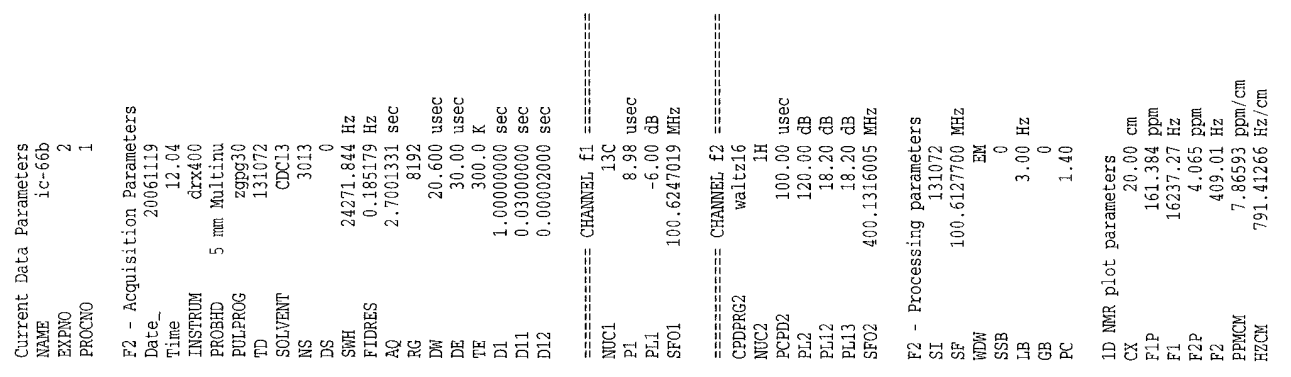

$\mp g Z \cdot 2$

$\left\llcorner\subseteq \varepsilon^{\circ} \tau \varepsilon\right.$

हOح'

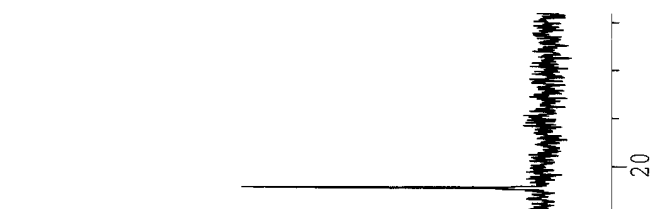

$667 \cdot$ โ9

0 T5. $89 \longrightarrow$

$700^{\circ} \mathrm{LL}$

TZE $L L$

$\hbar \hbar \overline{7} \cdot 2 Z L$

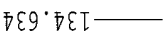

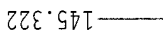

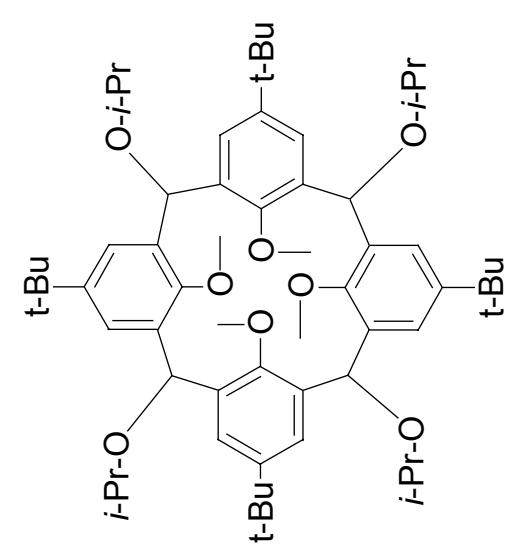

$\tau 6 \tau^{\varepsilon} \varsigma \tau$

ưdd

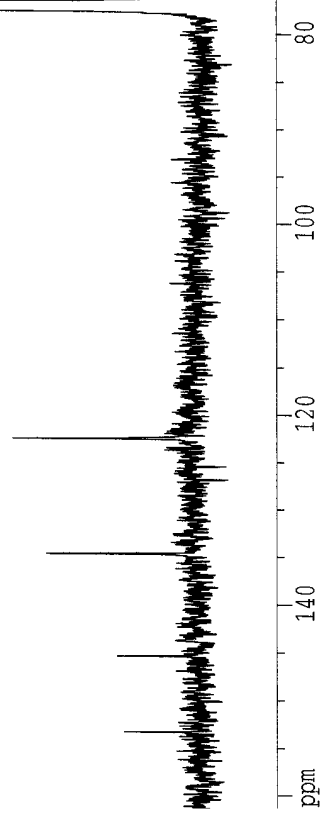


Figure S10. ${ }^{1} \mathrm{H}$ NMR spectrum of $\mathbf{6} f$ in $\mathrm{CDCl}_{3}$.
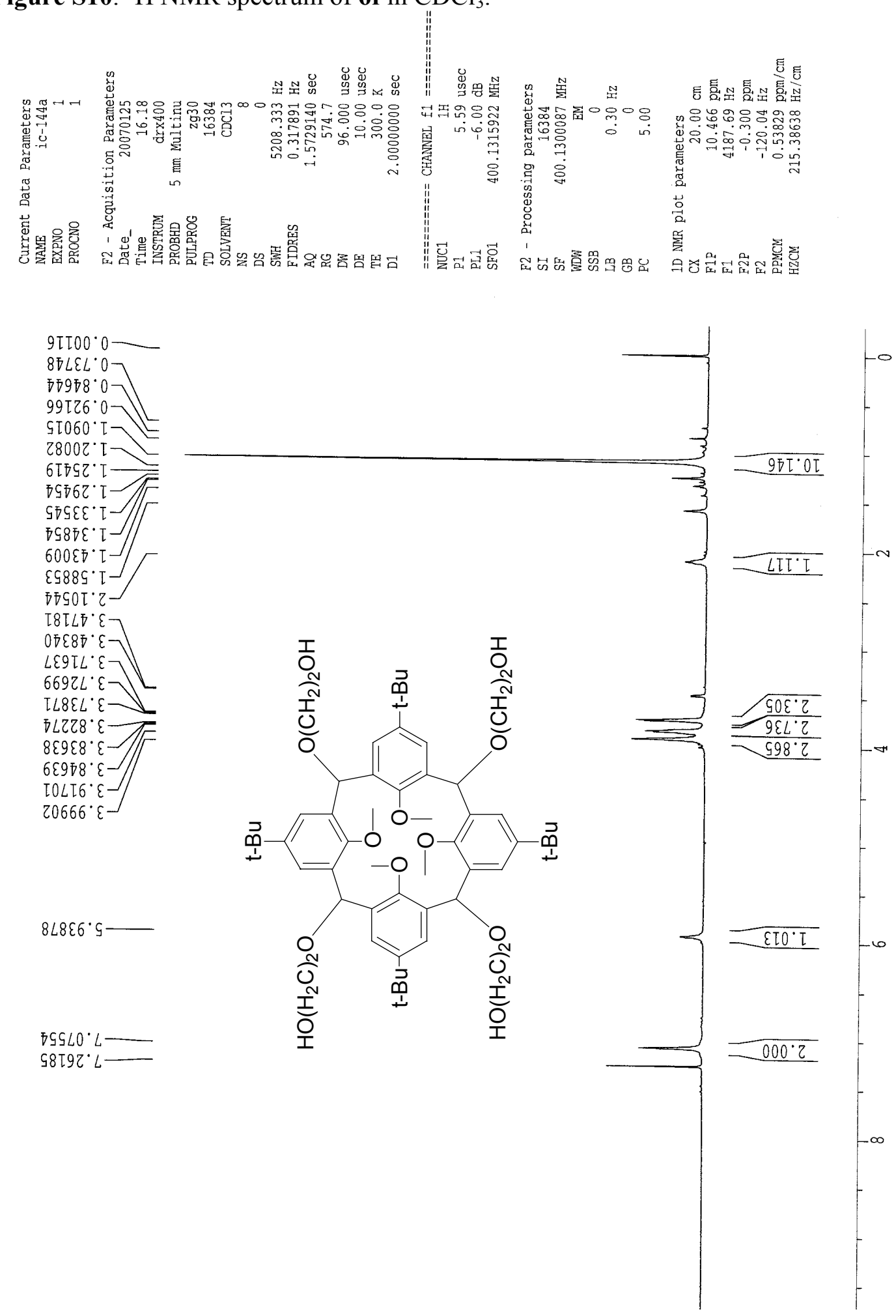
Figure S11. ${ }^{13} \mathrm{C}$ NMR spectrum of $\mathbf{6} f$ in $\mathrm{CDCl}_{3}$.

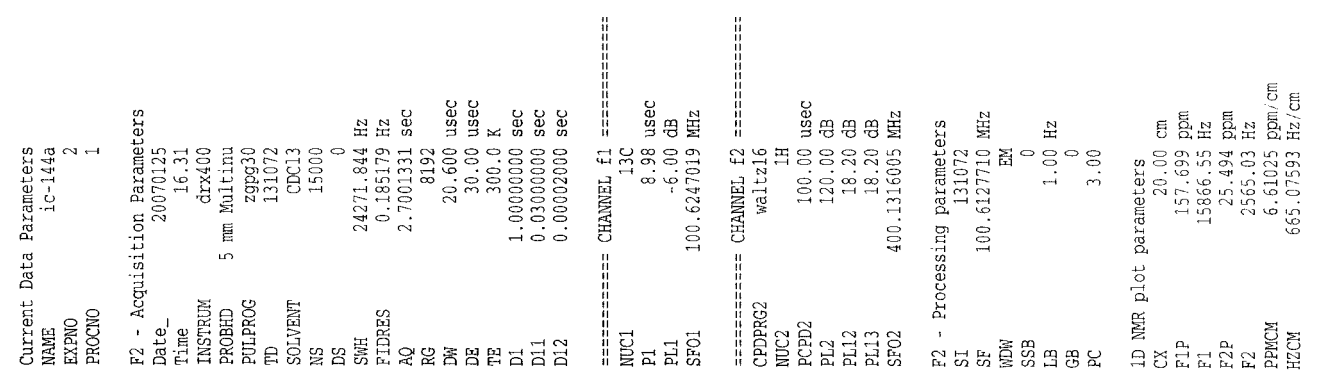

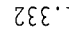

$9 \varepsilon \tau \cdot \nabla \varepsilon-$

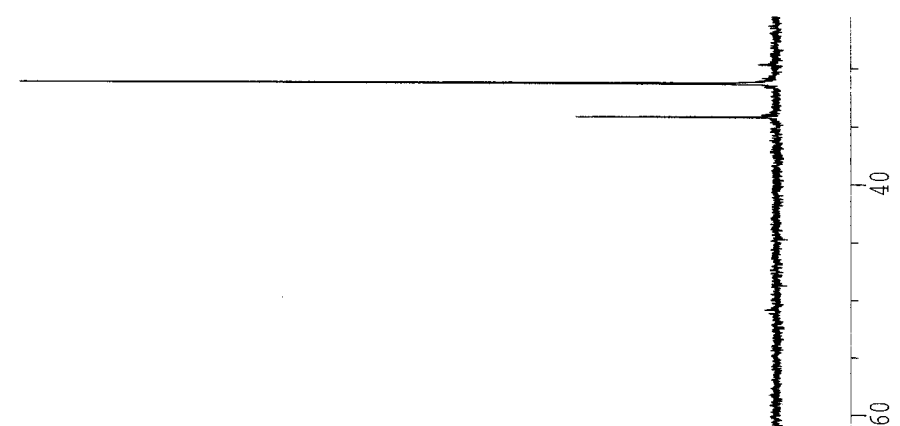

$999^{\circ} .99 \longrightarrow$
$780^{\circ} 29 \longrightarrow$

$\varepsilon S L \cdot O L$
$08 Z^{\circ} \varepsilon L$

$082^{\circ} \varepsilon L$

$666^{\circ} 9 \mathrm{~L}$

$9 T \varepsilon^{\circ} L L$

$68 T^{\circ}$ ZZT

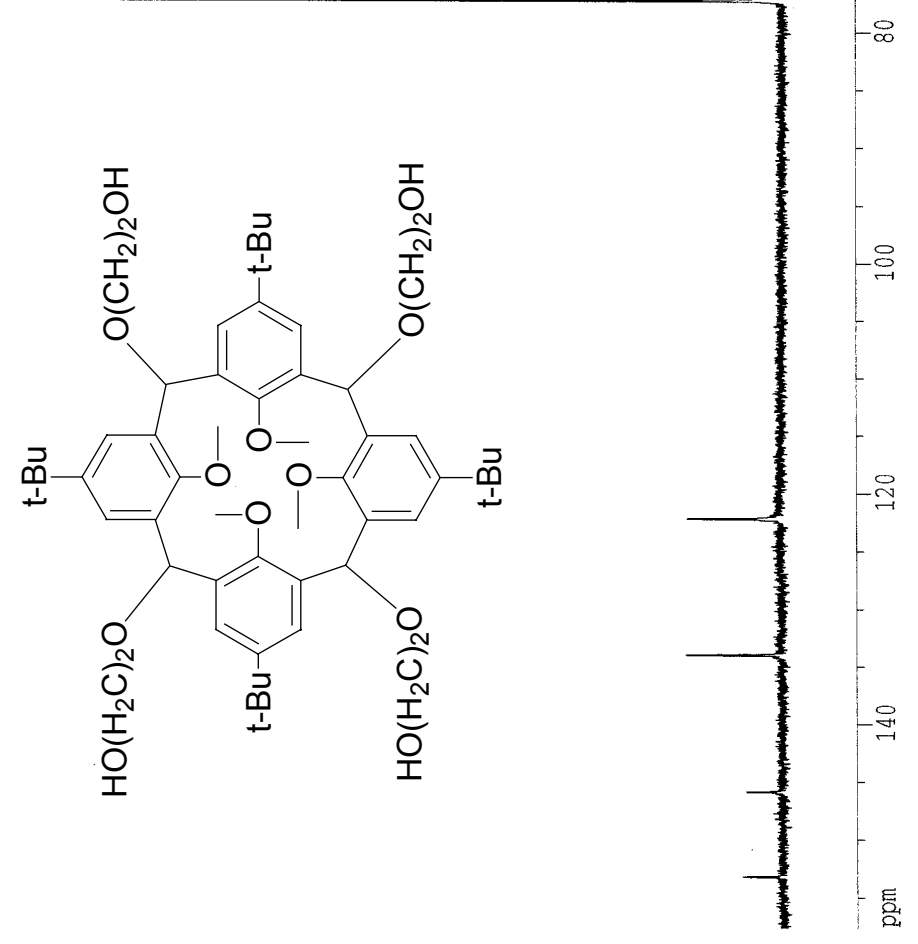

$\varepsilon \tau \sigma^{\circ} \varsigma 5 T-\ldots$

$066^{\circ} \varepsilon \varepsilon T-$

$96 T^{\circ} \varepsilon 9 T$
udd 
Figure S12. ${ }^{1} \mathrm{H}$ NMR spectrum of 8 in $\mathrm{CDCl}_{3}$.

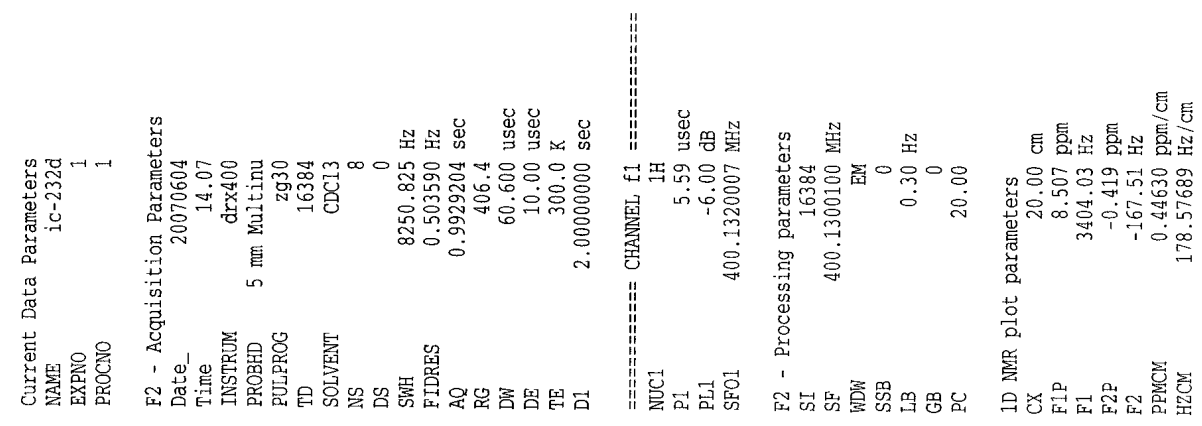

$1200^{\circ} 0^{-}$

$2268^{\circ} 0$

$9260^{\circ}$

$\varepsilon 062^{\circ} \mathrm{I}$

8TOE.

6095.

LLLT'Z -
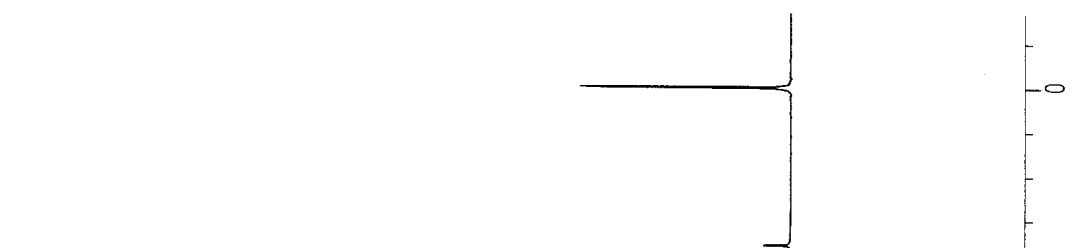

I766 $66^{\circ} \varepsilon$

$0280^{\circ}=$

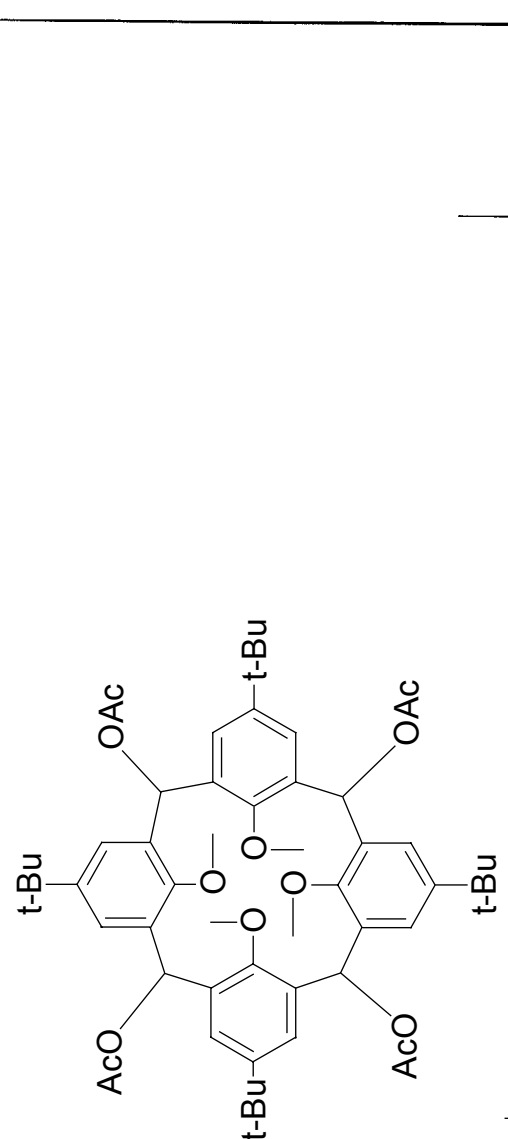

$=0622^{\circ}$

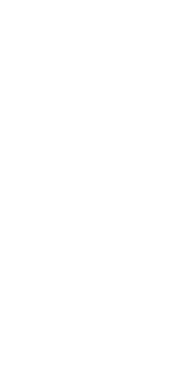

$\varepsilon 770^{\circ} \mathrm{L}$

$\angle 89 Z^{\circ} \mathrm{L}$

$+$

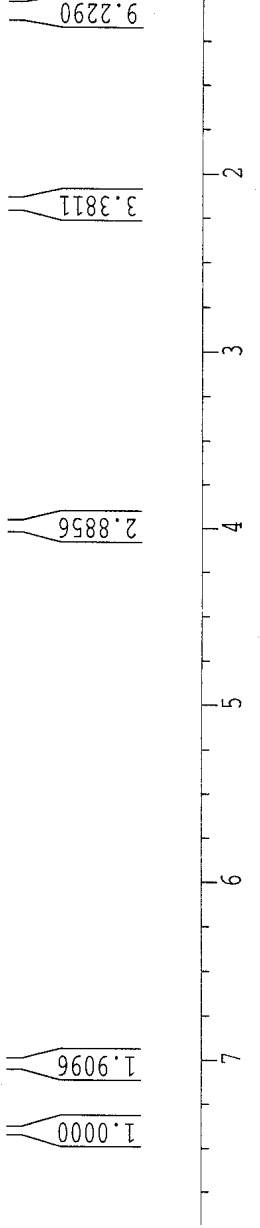


Figure S13. ${ }^{13} \mathrm{C}$ NMR spectrum of $\mathbf{8}$ in $\mathrm{CDCl}_{3}$.
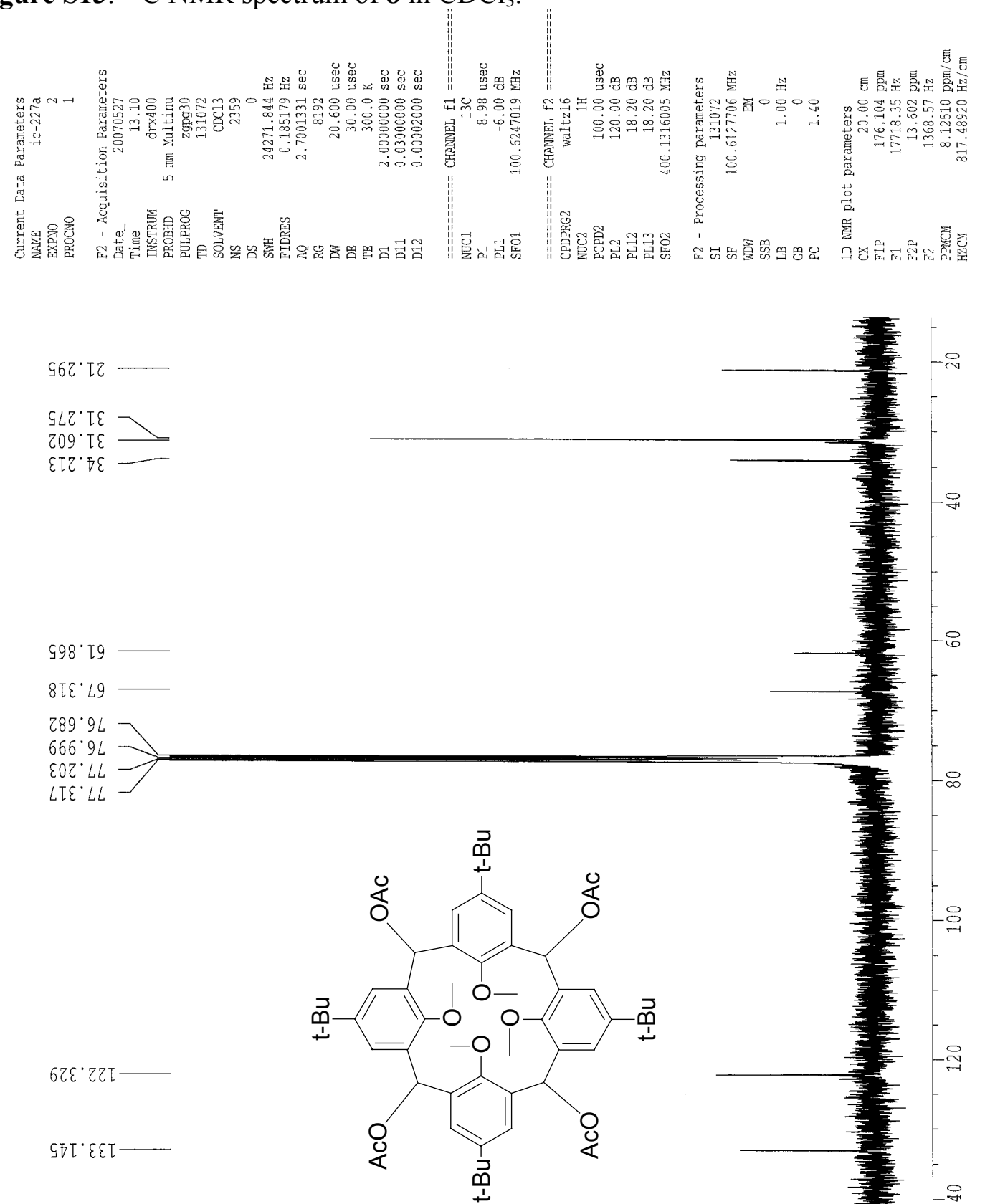

$279^{\circ} 97$

†५ร' $29 T$

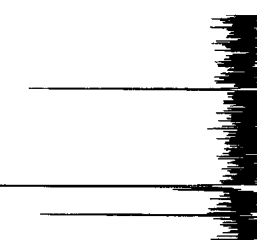


Figure S14. ${ }^{1} \mathrm{H}$ NMR spectrum of 9 in acetone- $\mathrm{d}_{6}$.
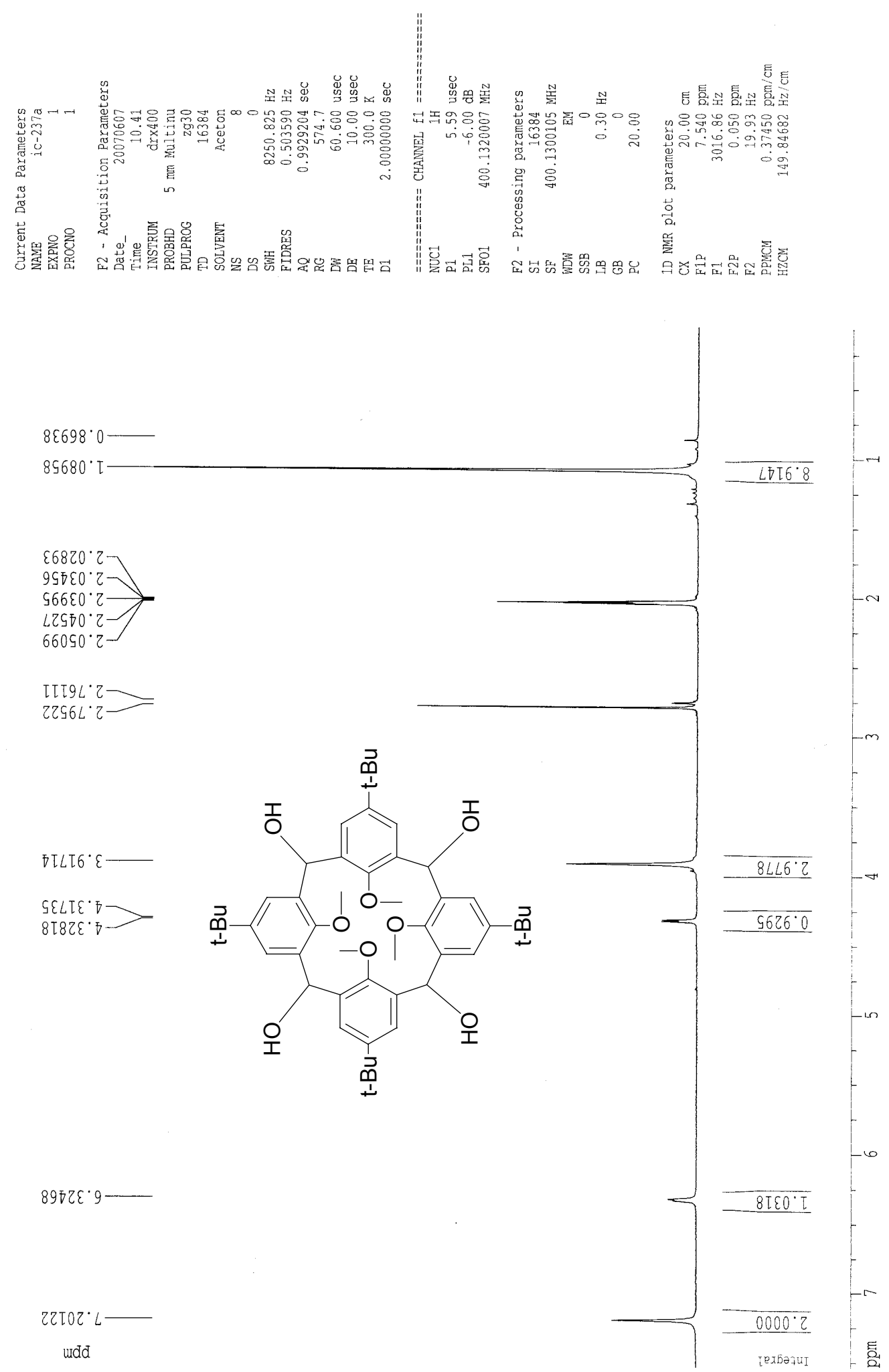
Figure S15. ${ }^{13} \mathrm{C}$ NMR spectrum of 9 in acetone- $\mathrm{d}_{6}$.

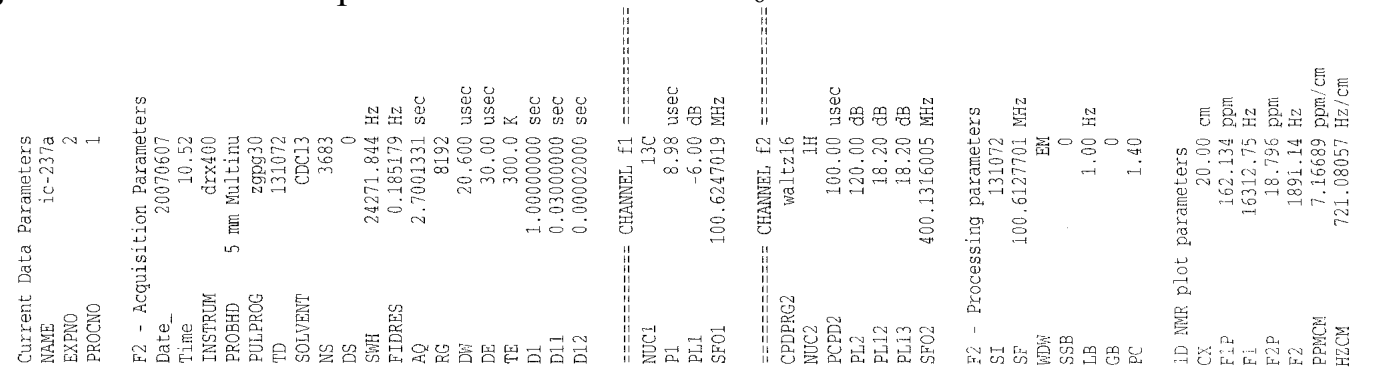

$\angle 60^{\circ} .9$

$\varepsilon \subseteq \nabla^{\circ} 79$

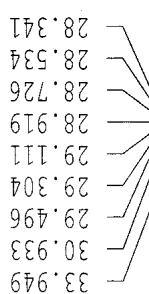

OSE. TZT -

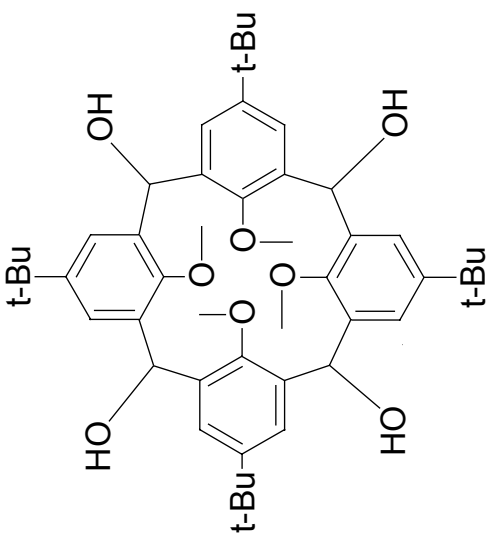

DIT'LEI-

TEE. EET -

8TS'29T

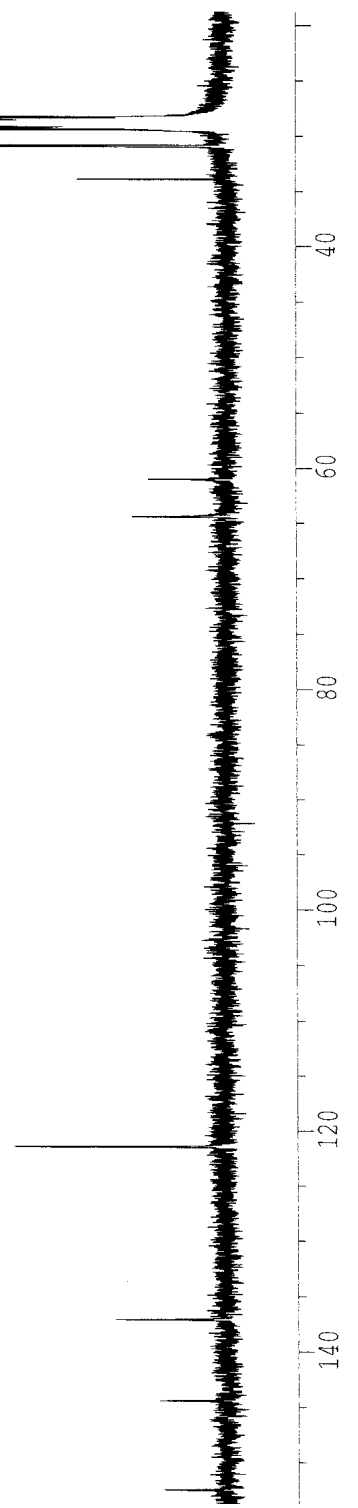


Figure S16. ${ }^{1} \mathrm{H}$ NMR spectrum of $\mathbf{1 0}$ in $\mathrm{CDCl}_{3}$
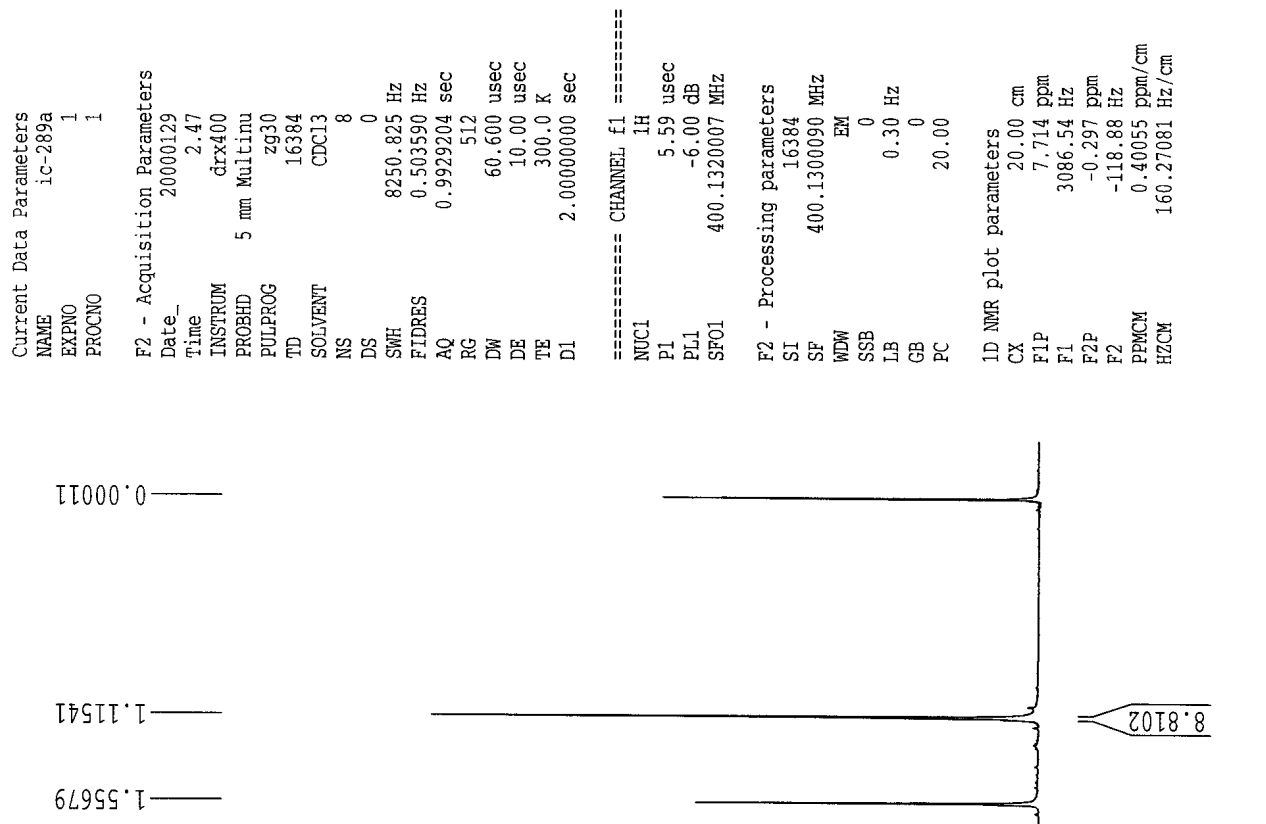

$97850^{\circ} 5$

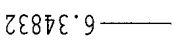

LLL9T:LD
89T9Z:LL uđđd
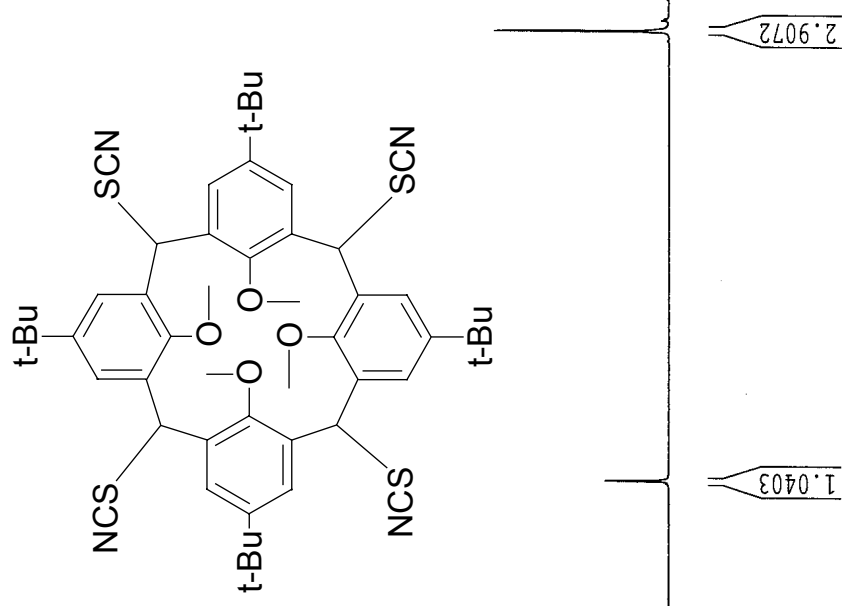

$>2018 \cdot 8$

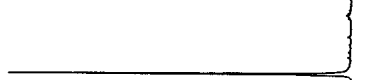

2018.8

$\widehat{\operatorname{EOT0} 0^{\circ}}$

$=0000^{\circ}$

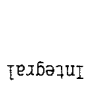


Figure S17. ${ }^{13} \mathrm{C}$ NMR spectrum of $\mathbf{1 0}$ in $\mathrm{CDCl}_{3}$.

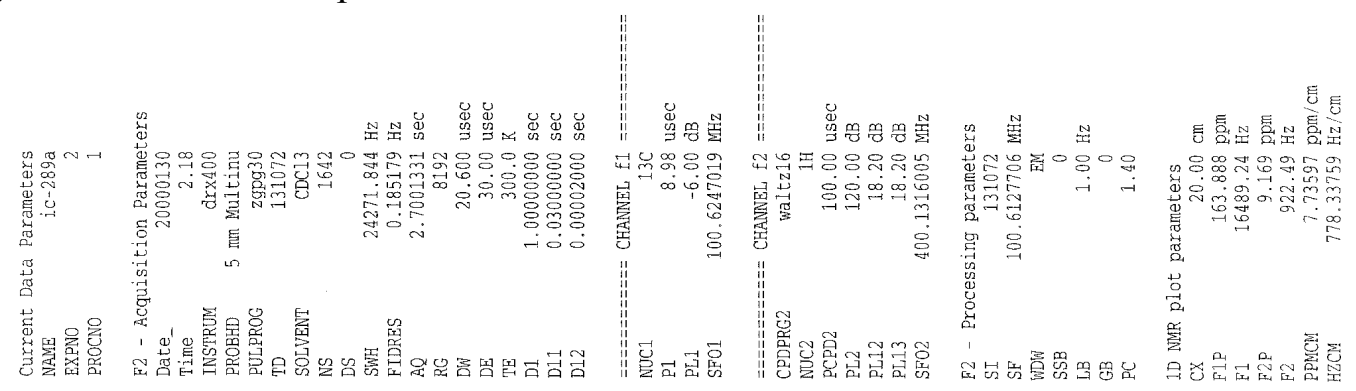

$\nabla L 0^{\circ} T \varepsilon$

6TS. โ⿺

$\mp 90^{\circ} \subseteq 7-$

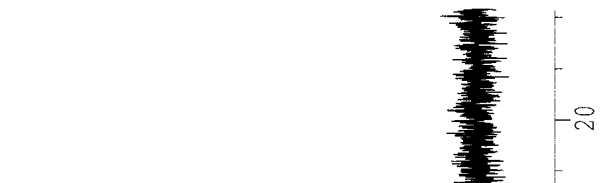

$579 \cdot 79$

$289^{\circ} 9 L$
$000^{\circ} L L$

$000^{\circ} L L$

LIE LL

$60 L ' L L$

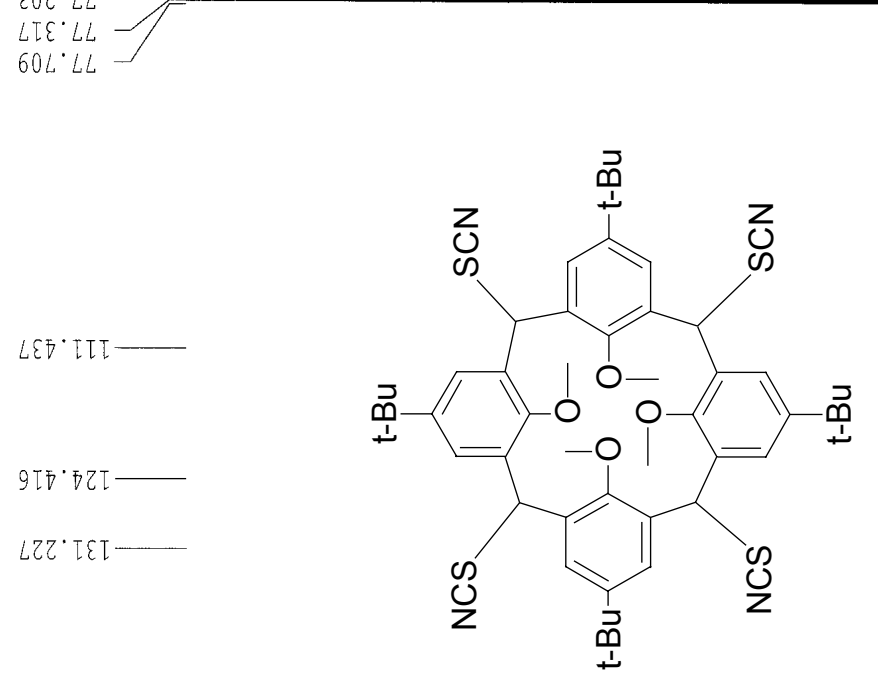

$\varepsilon 6 \Phi^{\circ} \angle \bar{L}$

५ร8. $25 T$

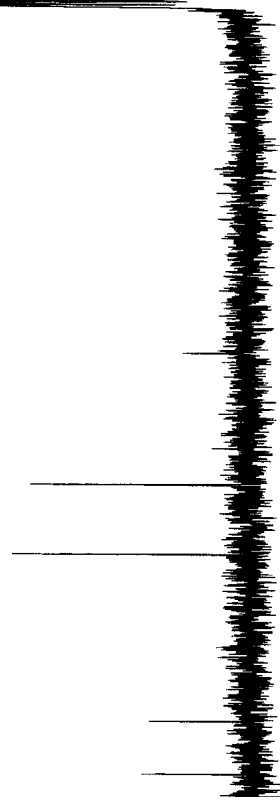


Figure S18. ${ }^{1} \mathrm{H}$ NMR spectrum of 11 in $\mathrm{CDCl}_{3}$

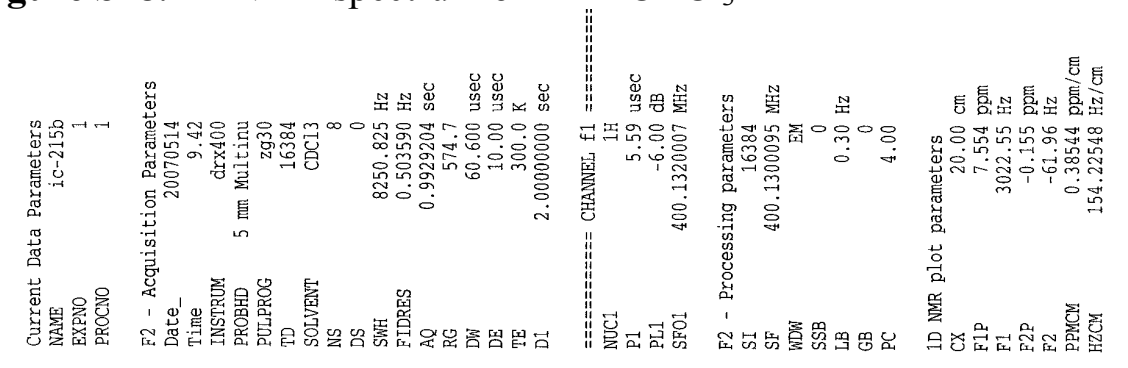

$2000^{\circ} 0^{-}$

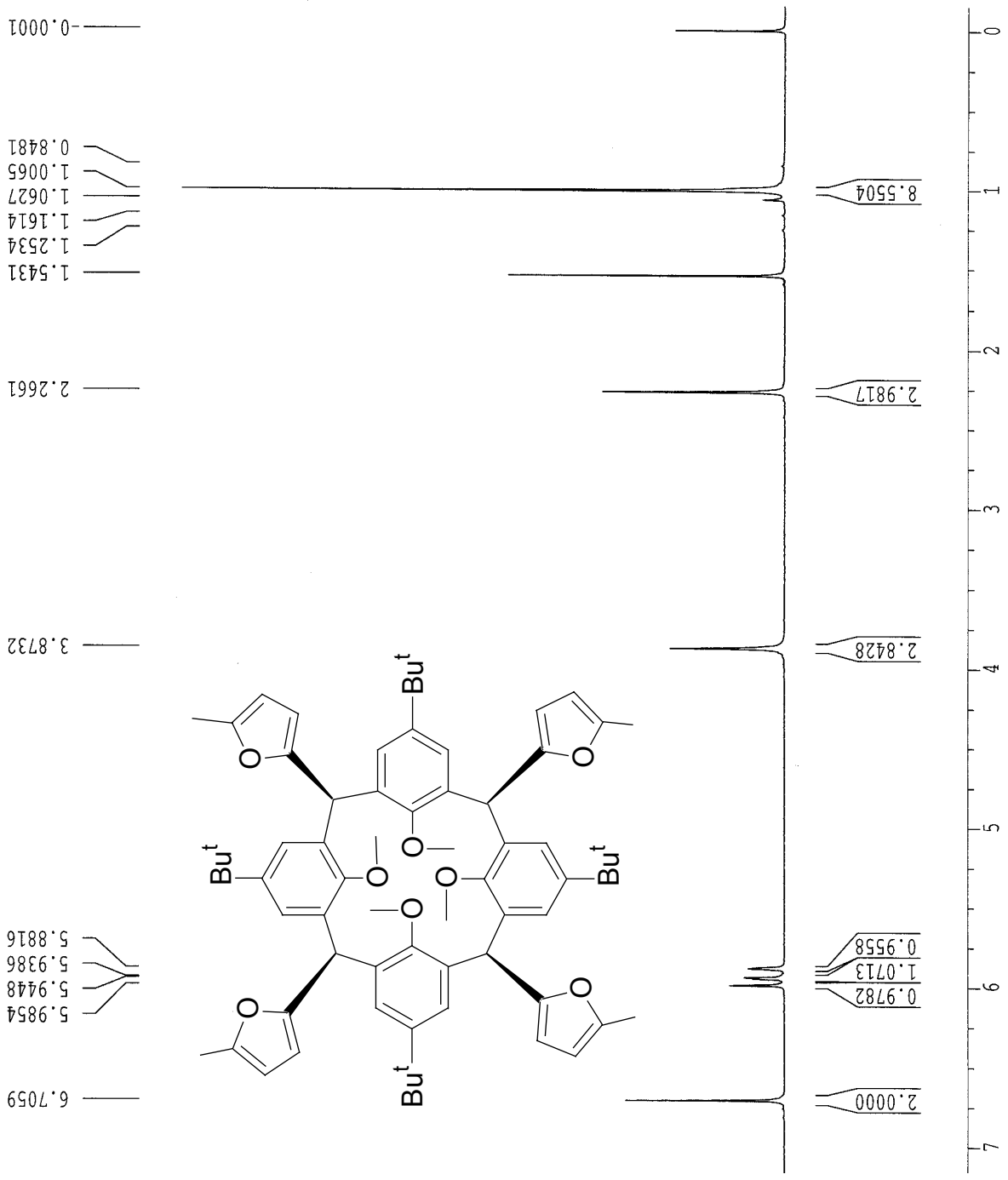


Figure S19. ${ }^{13} \mathrm{C}$ NMR spectrum of 11 in $\mathrm{CDCl}_{3}$.

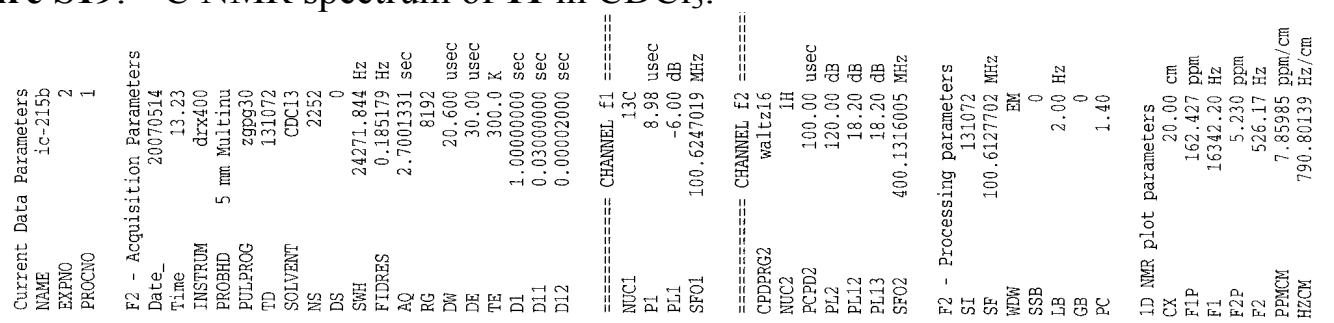

$079^{\circ} \varepsilon T$

$\varepsilon 9 \tau^{\circ} \cdot \varepsilon-$

$\angle 00^{\circ} \overline{7}$

$08 L^{\circ} L \varepsilon$

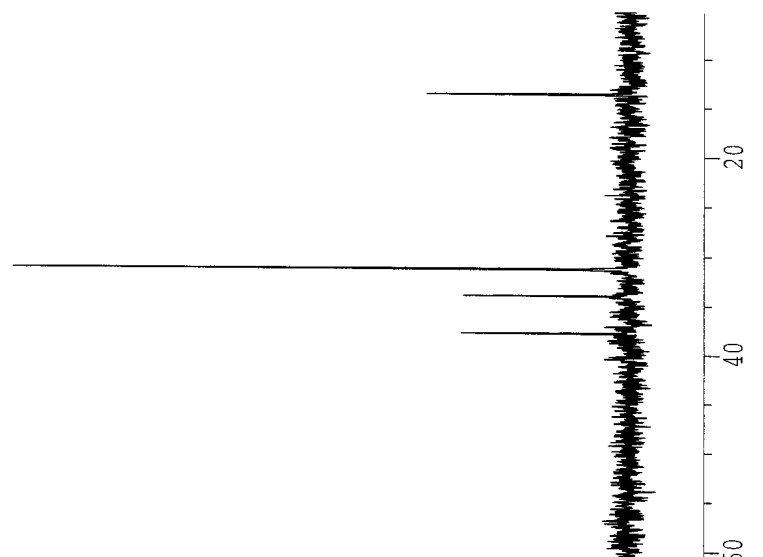

$968 \cdot 19$

$\varepsilon 89^{\circ} \cdot 9 L$

$807^{\circ} L L$

8IE'LL

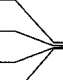


Figure S20. ${ }^{1} \mathrm{H}$ NMR spectrum of 12 in $\mathrm{CDCl}_{3}$
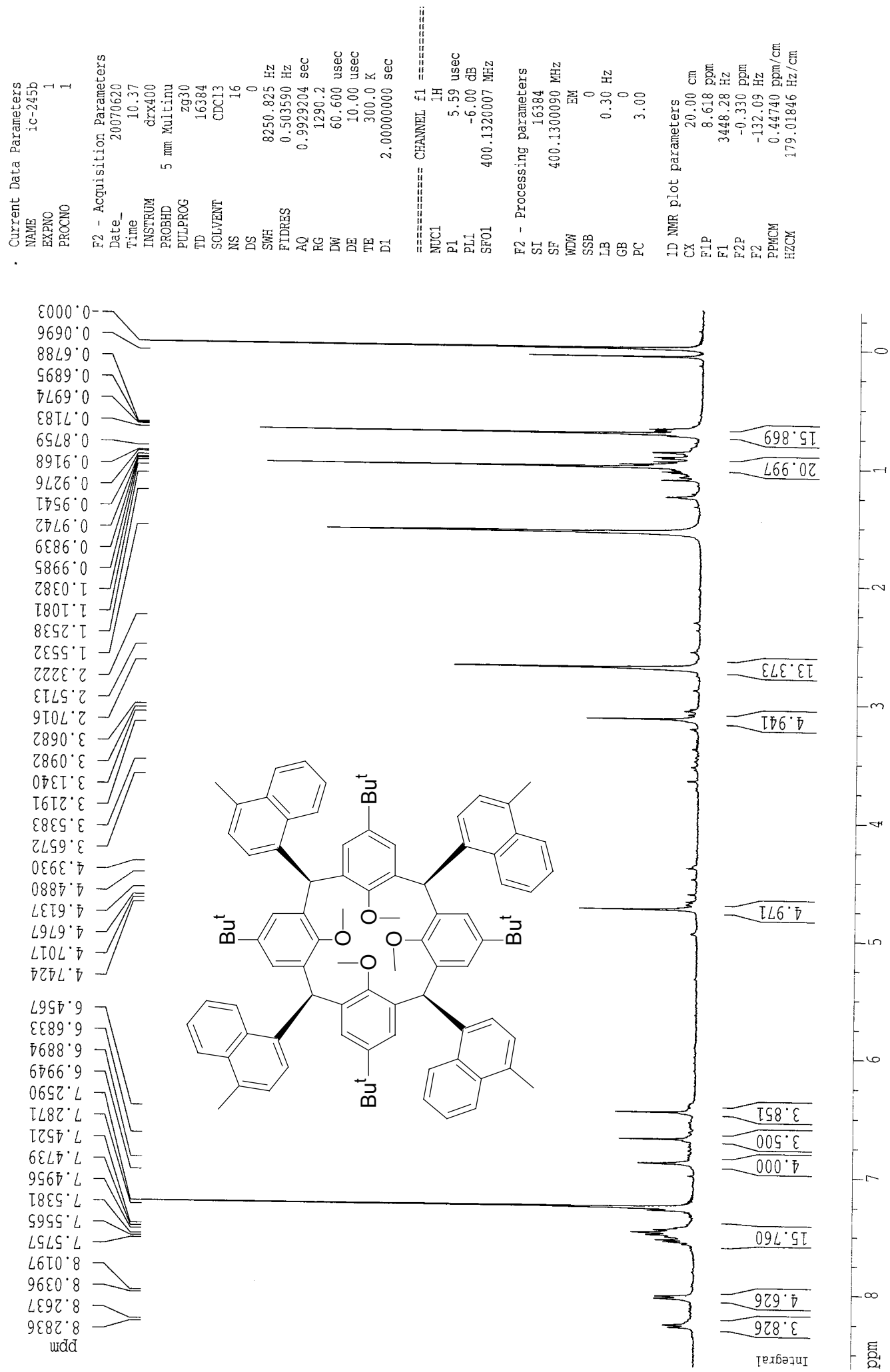
Figure S21. ${ }^{13} \mathrm{C}$ NMR spectrum of $\mathbf{1 2}$ in $\mathrm{CDCl}_{3}$.
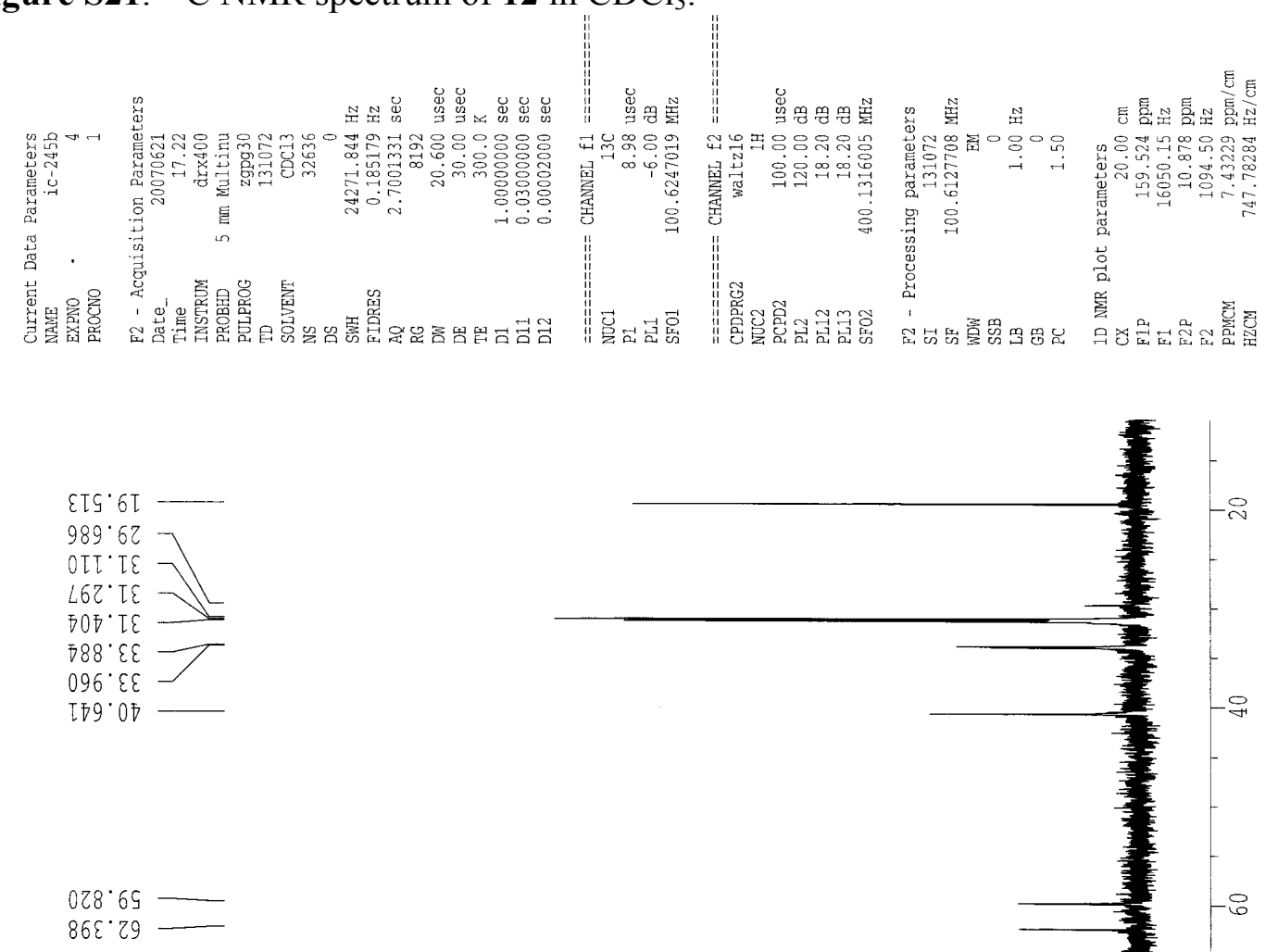

$078 \cdot 69-$

$86 \varepsilon^{\circ} 29$

$699^{\circ} 9 L$
$\angle 86.9 L$
$06 T^{\circ} L L$

$70 \varepsilon^{\circ} L L$
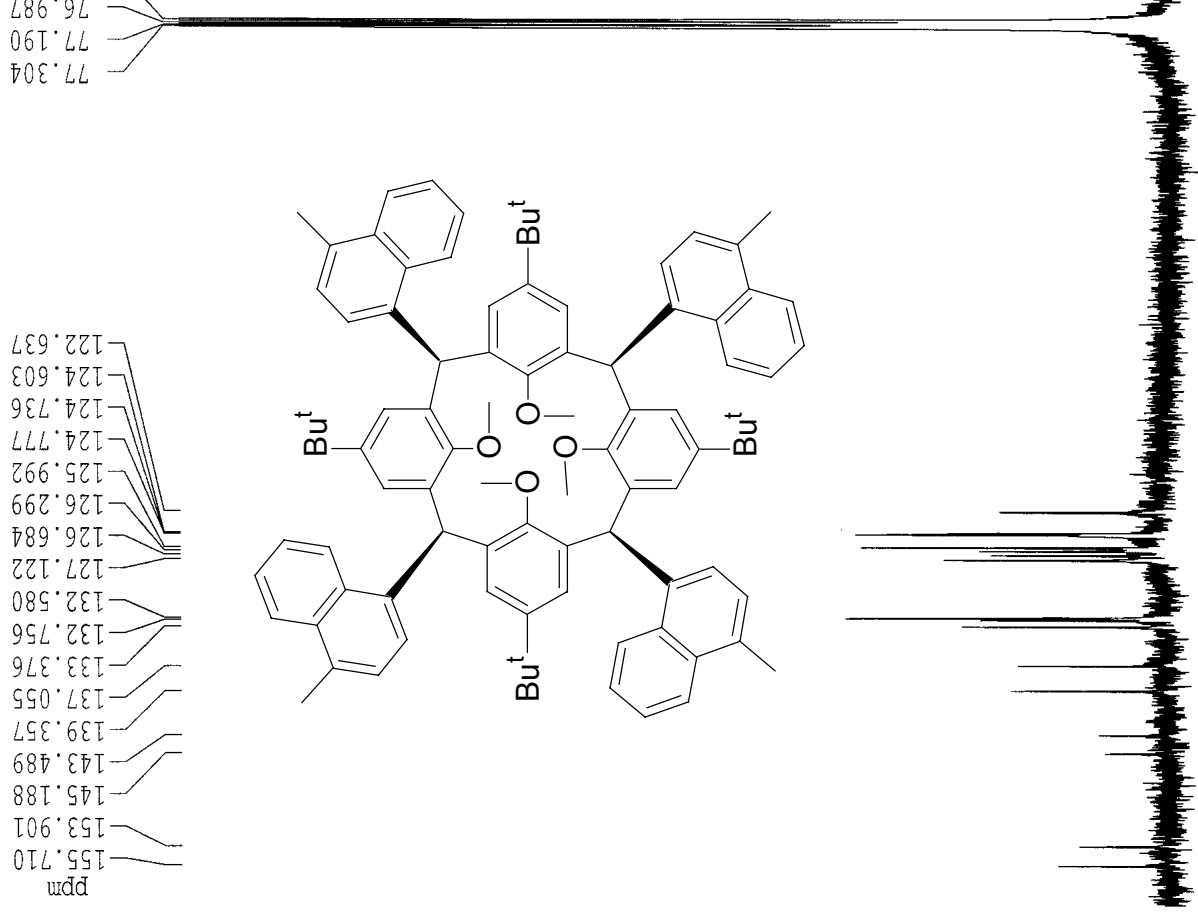
Figure S22. ${ }^{1} \mathrm{H}$ NMR spectrum of 14 in $\mathrm{CDCl}_{3}$

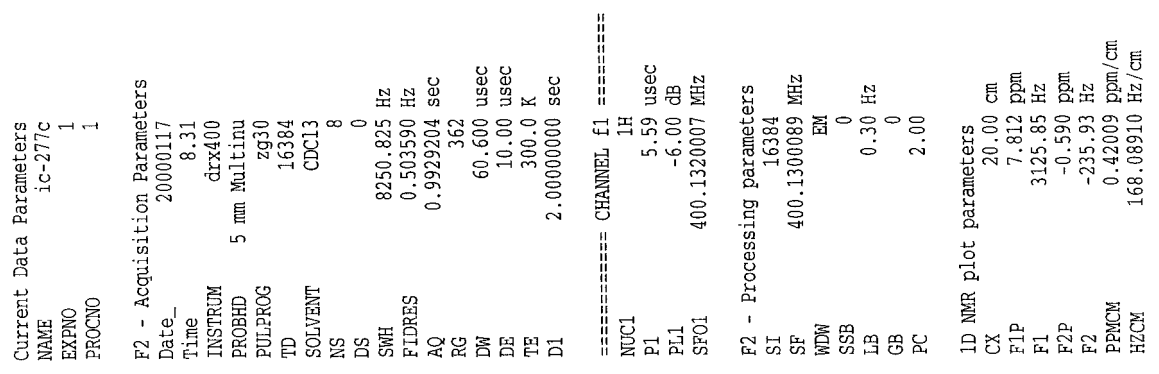

$\varepsilon L \varepsilon 00^{\circ} 0$
$\varepsilon 2 \varepsilon L 0^{\circ} 0-$
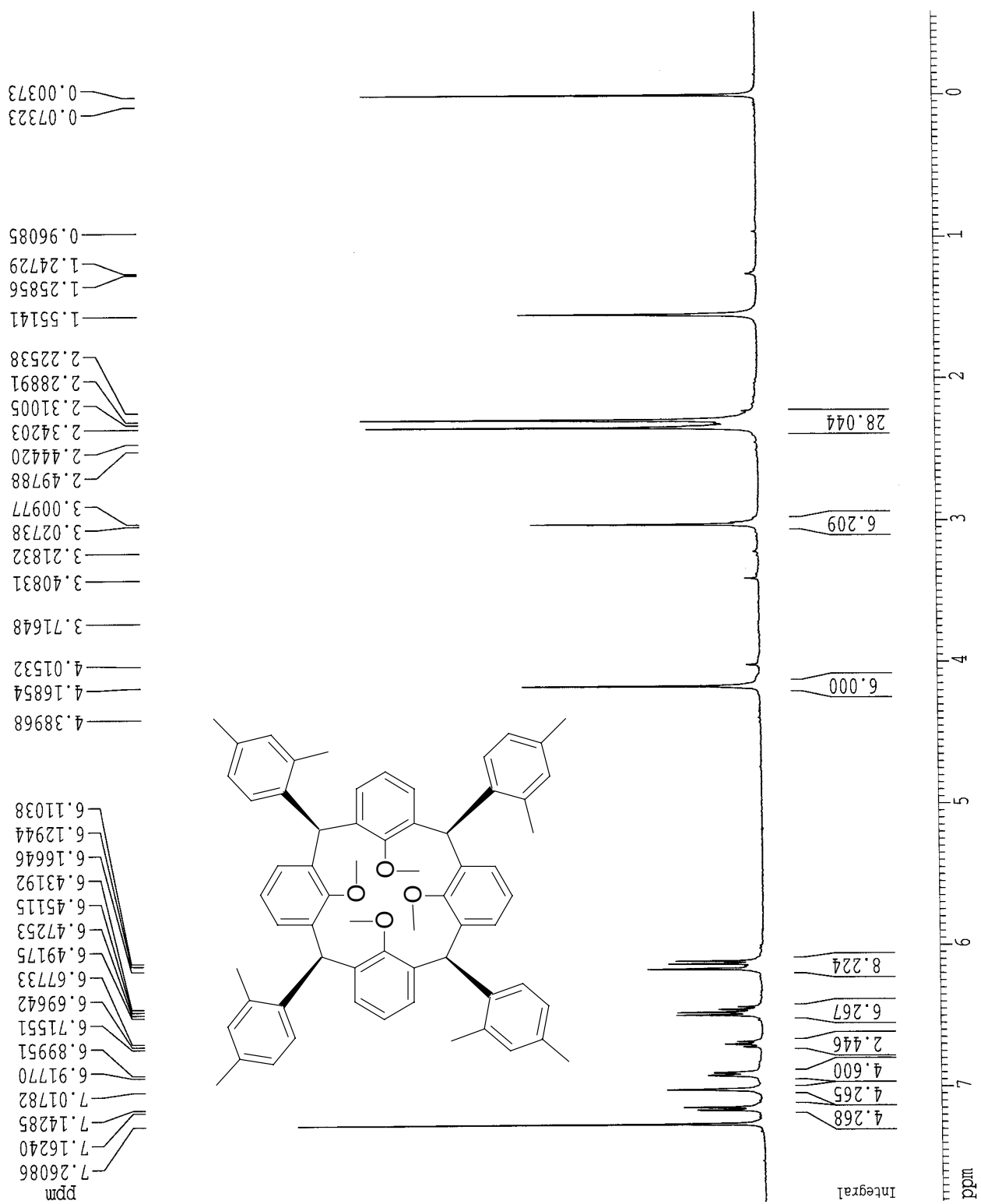
Figure S23. ${ }^{13} \mathrm{C}$ NMR spectrum of $\mathbf{1 4}$ in $\mathrm{CDCl}_{3}$.

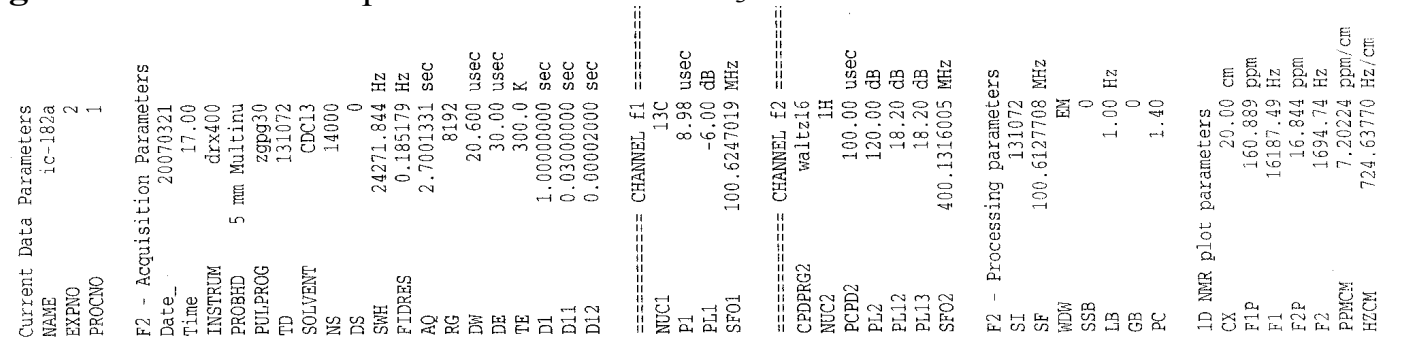

$79 \varepsilon^{\circ} 02$

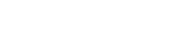

$889^{\circ} 62$

$227 \cdot 07$

$687.09=$
$62 L \cdot 19=$

$8 L 9 \cdot 9 L$
$966 \cdot 9 L$

$966.9 L$

$66 T^{\circ} L L$
$\varepsilon T \varepsilon^{\circ} L L$

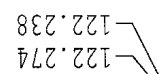

ZSL'SZT-

t2Z.9ZT

$050^{\circ} 621-$

227.621

$06 T^{\circ}$ TET -

$06 \varepsilon^{\circ} \mathrm{c} \varepsilon \mathrm{T}$

I8I. รET-

otg. $9 \varepsilon T-$

$790^{\circ}$ LET-

$\varepsilon 06^{\circ} 6 \varepsilon \tau-$
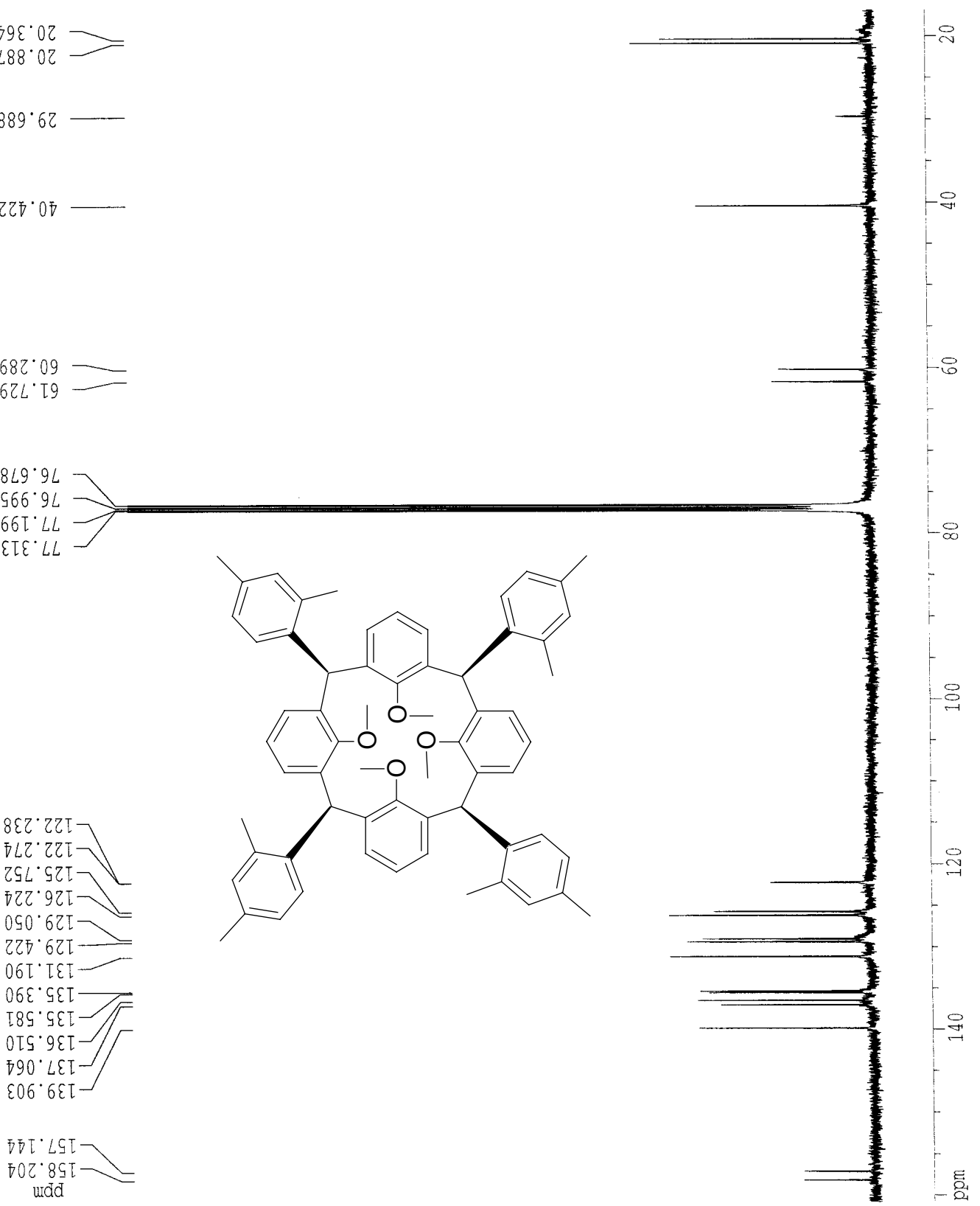


\section{$R c c c$ isomer of $\mathbf{1 5}$, cone conformation (tetraequatorial)}

This structure has $34.4430 \mathrm{Kcal} / \mathrm{mol}$.
0 imaginary frequencies. Final steric energy is

\begin{tabular}{|c|c|c|c|c|c|c|}
\hline \multicolumn{2}{|c|}{ COORDINATES } & ATED TO & $\mathrm{N}$ & $h$ & T & \\
\hline C ( & 1) & -3.08293 & -1.34744 & .24939 & 2) & 2) \\
\hline Ci & 2) & -2.78957 & -.09991 & -.33534 & 2) & 2) \\
\hline Ci & 3) & -3.21821 & 1.09448 & .27670 & 2) & 2) \\
\hline Ci & 4) & -4.09568 & 1.01550 & 1.36770 & 2) & 2) \\
\hline C ( & 5) & -4.48142 & -.21974 & 1.88208 & ( & 2) \\
\hline Ci & 6) & -3.95883 & -1.39227 & 1.34301 & ( & 2) \\
\hline C ( & 7) & -1.36240 & 2.81000 & .47212 & ( & 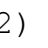 \\
\hline Ci & 8) & -.14774 & 2.78185 & -.23969 & ( & 0 \\
\hline C ( & 9) & 1.07853 & 2.89575 & .44368 & ( & 2) \\
\hline Ci & 10) & 1.06681 & 3.23489 & 1.80319 & ( & 2) \\
\hline C ( & 11) & -.13674 & 3.37636 & 2.48955 & ( & 2) \\
\hline Ci & 12) & -1.34240 & 3.14087 & 1.83384 & ( & 2) \\
\hline C ( & 13) & 3.08294 & 1.34743 & .24938 & ( & 2 \\
\hline C ( & 14) & 2.78956 & .09993 & -.33534 & ( & 2) \\
\hline C ( & 15) & 3.21823 & -1.09449 & .27670 & ( & 2) \\
\hline Ci & 16) & 4.09571 & -1.01551 & 1.36767 & ( & 2) \\
\hline C ( & 17) & 4.48147 & .21974 & 1.88204 & ( & 2 \\
\hline C ( & 18) & 3.95888 & 1.39225 & 1.34299 & ( & 2) \\
\hline Ci & 19) & 1.36242 & -2.81001 & .47214 & ( & 2) \\
\hline C ( & $20)$ & .14770 & -2.78185 & -.23969 & ( & 2) \\
\hline C ( & 21) & -1.07851 & -2.89575 & .44365 & ( & 2) \\
\hline C ( & 22) & -1.06679 & -3.23493 & 1.80321 & ( & 2) \\
\hline Ci & 23) & .13672 & -3.37638 & 2.48954 & 2 & 2) \\
\hline C ( & 24) & 1.34242 & -3.14086 & 1.83381 & ( & 2) \\
\hline 01 & 25) & -.14837 & 2.38998 & -1.54660 & 6 & 6) \\
\hline Ci & 26) & -2.68143 & 2.43938 & -.17776 & ( & 1) \\
\hline 01 & 27) & -1.86314 & -.04016 & -1.33467 & 6 & 6) \\
\hline C ( & 28) & -2.40227 & -2.61241 & -.23873 & 1 & 1) \\
\hline 0( & 29) & .14833 & -2.38996 & -1.54660 & 6 & 6) \\
\hline C ( & $30)$ & 2.68144 & -2.43938 & -.17776 & ( & 1) \\
\hline O( & 31) & 1.86311 & .04018 & -1.33464 & 6 & 6) \\
\hline C ( & 32) & 2.40227 & 2.61241 & -.23872 & 1 & 1) \\
\hline $\mathrm{H}($ & 33) & -4.46505 & 1.93264 & 1.85552 & ( & 5) \\
\hline $\mathrm{H}($ & $34)$ & -4.21971 & -2.35635 & 1.81018 & 5 & 5) \\
\hline H ( & 35) & 2.01481 & 3.36151 & 2.35137 & ( & 5) \\
\hline H ( & $36)$ & -2.28368 & 3.18995 & 2.40596 & ( & 5) \\
\hline $\mathrm{H}($ & $37)$ & 4.46508 & -1.93265 & 1.85549 & ( & 5) \\
\hline $\mathrm{H}($ & 38) & 4.21980 & 2.35634 & 1.81014 & ( & 5) \\
\hline H ( & 39) & -2.01479 & -3.36161 & 2.35138 & ( & 5) \\
\hline $\mathrm{H}($ & $40)$ & 2.28370 & -3.18991 & 2.40593 & ( & 5) \\
\hline $\mathrm{H}($ & $41)$ & -2.56629 & 2.40531 & -1.28634 & ( & 5) \\
\hline H ( & $42)$ & -2.22494 & -2.52343 & -1.33496 & ( & 5) \\
\hline $\mathrm{H}($ & 43) & 2.56627 & -2.40530 & -1.28633 & ( & 5) \\
\hline $\mathrm{H}($ & 44) & 2.22492 & 2.52344 & -1.33495 & ( & 5) \\
\hline C ( & 45) & -.15142 & 3.45829 & -2.48172 & ( & 1) \\
\hline C ( & 46) & .15140 & -3.45826 & -2.48174 & ( & 1) \\
\hline
\end{tabular}




\begin{tabular}{|c|c|c|c|c|}
\hline 47) & -2.39911 & -.05761 & -2.64980 & 1) \\
\hline 48$)$ & 2.39905 & .05763 & -2.64978 & 1) \\
\hline 49$)$ & -.12812 & 3.04368 & -3.51010 & 5) \\
\hline 50$)$ & -1.07307 & 4.07780 & -2.37366 & 5) \\
\hline 51) & .74322 & 4.11180 & -2.34491 & 5) \\
\hline 52) & .12811 & -3.04364 & -3.51010 & 5) \\
\hline 53) & 1.07305 & -4.07776 & -2.37367 & 5) \\
\hline 54$)$ & -.74323 & -4.11178 & -2.34494 & 5) \\
\hline 55) & -3.06688 & .81985 & -2.82141 & 5) \\
\hline 56) & -1.56844 & -.01321 & -3.38340 & 5) \\
\hline 57) & -2.98009 & -.99267 & -2.83298 & 5) \\
\hline 58$)$ & 1.56836 & .01322 & -3.38336 & 5) \\
\hline 59) & 2.98003 & .99268 & -2.83299 & 5) \\
\hline 60) & 3.06682 & -.81984 & -2.82141 & 5) \\
\hline 61) & -5.15947 & -.26705 & 2.75124 & 5) \\
\hline 62) & -.13274 & 3.62518 & 3.56440 & 5) \\
\hline 63) & 5.15954 & .26705 & 2.75118 & 5) \\
\hline 64$)$ & .13273 & -3.62522 & 3.56439 & 5) \\
\hline 65) & -3.23292 & -3.74311 & -.01231 & 6) \\
\hline 66) & -3.65170 & 3.42897 & .13774 & 6) \\
\hline 67) & 3.23293 & 3.74310 & -.01231 & 6) \\
\hline 68) & 3.65171 & -3.42897 & .13771 & 6) \\
\hline 69) & -3.43186 & 4.66948 & -.51368 & 1) \\
\hline 70$)$ & 4.29849 & 3.84347 & -.94428 & 1) \\
\hline 71) & 3.43185 & -4.66948 & -.51371 & 1) \\
\hline 72$)$ & -4.29850 & -3.84347 & -.94427 & 1) \\
\hline 73$)$ & -2.44330 & 5.10313 & -.23126 & 5) \\
\hline 74$)$ & -3.48264 & 4.54683 & -1.62210 & 5) \\
\hline 75$)$ & -4.22231 & 5.38506 & -.20889 & 5) \\
\hline 76) & 4.92795 & 4.71893 & -.68513 & 5) \\
\hline 77) & 4.94121 & 2.93160 & -.92146 & 5) \\
\hline 78$)$ & 3.90397 & 3.99183 & -1.97833 & 5) \\
\hline 79) & 2.44329 & -5.10313 & -.23129 & 5) \\
\hline 80$)$ & 3.48262 & -4.54682 & -1.62213 & 5) \\
\hline 81) & 4.22231 & -5.38507 & -.20894 & 5) \\
\hline 82$)$ & -4.94122 & -2.93161 & -.92141 & 5) \\
\hline 83$)$ & -3.90400 & -3.99181 & -1.97832 & 5) \\
\hline 84$)$ & -4.92795 & -4.71894 & -.68511 & 5) \\
\hline
\end{tabular}




\section{$R c c c$ isomer of 15, partial cone conformation (diequatorial-diisoclinal (I))}

This structure has $38.7987 \mathrm{Kcal} / \mathrm{mol}$
0 imaginary frequencies, final steric energy is

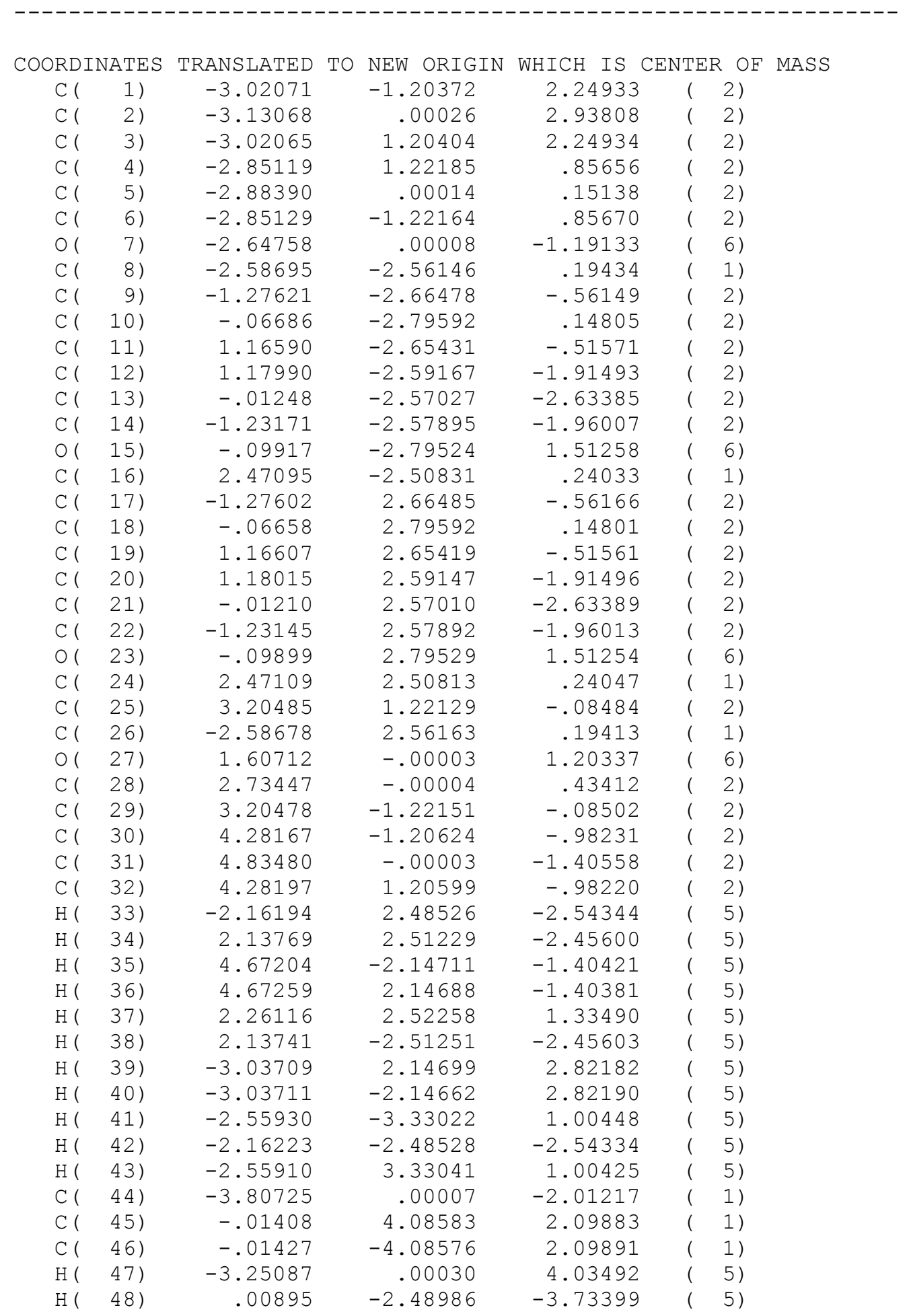




\begin{tabular}{|c|c|c|c|c|}
\hline 49) & .00937 & 2.48963 & -3.73402 & ( \\
\hline 50) & 5.66697 & -.00005 & -2.13012 & ( \\
\hline 51) & -4.43310 & -.90345 & -1.82959 & 1 \\
\hline 52) & -4.43307 & .90361 & -1.82966 & ( \\
\hline 53) & -3.50072 & .00002 & -3.07800 & ( \\
\hline 54) & .94757 & 4.58369 & 1.82775 & ( \\
\hline 55) & -.86213 & 4.73126 & 1.76687 & ( \\
\hline 56) & -.05877 & 3.98968 & 3.20277 & ( \\
\hline 57) & .94734 & -4.58366 & 1.82778 & ( \\
\hline 58$)$ & -.05887 & -3.98956 & 3.20286 & ( \\
\hline 59) & -.86237 & -4.73117 & 1.76704 & ( \\
\hline 60$)$ & 1.82557 & -.00010 & 2.60591 & ( \\
\hline 61) & 2.39290 & -.90757 & 2.92187 & ( \\
\hline 62$)$ & 2.39295 & .90731 & 2.92195 & ( \\
\hline 63) & .84684 & -.00010 & 3.12785 & ( \\
\hline 64$)$ & 2.26104 & -2.52273 & 1.33475 & ( \\
\hline 65) & 3.25767 & 3.64440 & -.09085 & ( \\
\hline 66) & 3.25745 & -3.64464 & -.09098 & ( \\
\hline 67) & -3.69609 & -2.85311 & -.64272 & ( \\
\hline 68$)$ & -3.69589 & 2.85331 & -.64296 & ( \\
\hline 69) & 4.21507 & -3.96333 & .90704 & ( \\
\hline 70$)$ & -3.89770 & -4.24421 & -.83971 & ( \\
\hline 71) & -3.89741 & 4.24441 & -.84002 & ( \\
\hline 72$)$ & 4.21524 & 3.96310 & .90722 & ( \\
\hline 73$)$ & 4.88950 & -3.09775 & 1.10960 & ( \\
\hline 74$)$ & 3.70861 & -4.26611 & 1.85508 & ( \\
\hline 75$)$ & 4.83501 & -4.81446 & .55903 & ( \\
\hline 76) & -4.16680 & -4.74221 & .12289 & ( \\
\hline 77) & -4.73446 & -4.39362 & -1.55204 & ( \\
\hline 78$)$ & -2.98615 & -4.72834 & -1.26354 & ( \\
\hline 79) & -4.16649 & 4.74248 & .12255 & ( \\
\hline 80$)$ & -2.98583 & 4.72846 & -1.26387 & ( \\
\hline 81$)$ & -4.73416 & 4.39383 & -1.55236 & ( \\
\hline 82$)$ & 3.70874 & 4.26596 & 1.85521 & ( \\
\hline 83$)$ & 4.88960 & 3.09748 & 1.10988 & ( \\
\hline 84$)$ & 4.83525 & 4.81417 & .55920 & ( \\
\hline
\end{tabular}




\section{Rcct isomer of 15, cone conformation (triequatorial-axial)}

This structure has 0 imaginary frequencies, final steric energy is $38.3381 \mathrm{Kcal} / \mathrm{mol}$.

\begin{tabular}{|c|c|c|c|c|c|c|}
\hline & TES & ATED & NEW & & & \\
\hline C ( & 1) & -3.27973 & -.98996 & .13204 & ( & 2) \\
\hline C ( & 2) & -2.79155 & .20065 & -.43918 & ( & 2) \\
\hline C ( & 3) & -3.15532 & 1.45086 & .09835 & ( & 2) \\
\hline $\mathrm{C}($ & 4) & -4.16801 & 1.49828 & 1.06732 & ( & 2) \\
\hline $\mathrm{C}($ & 5) & -4.75320 & .32677 & 1.54150 & ( & 1) \\
\hline C ( & 6) & -4.29347 & -.91015 & 1.09721 & ( & 2) \\
\hline $\mathrm{C}($ & 7) & -1.06562 & 2.84180 & .40705 & ( & 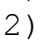 \\
\hline C ( & 8) & .15690 & 2.69597 & -.27579 & ( & . \\
\hline C ( & 9) & 1.36172 & 2.58096 & .44433 & ( & \\
\hline C ( & 10) & 1.35194 & 2.81545 & 1.82529 & ( & 1 \\
\hline C ( & 11) & .15640 & 3.07327 & 2.49213 & ( & \\
\hline C ( & 12) & -1.04706 & 3.06180 & 1.79106 & ( & \\
\hline $\mathrm{C}($ & 13) & 3.26090 & .88970 & .30054 & ( & \\
\hline C ( & 14) & 2.77042 & -.36017 & -.13103 & ( & 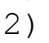 \\
\hline C ( & 15) & 3.14248 & -1.54021 & .54422 & ( & 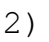 \\
\hline $\mathrm{C}($ & 16) & 4.15771 & -1.47398 & 1.51048 & ( & 21 \\
\hline C ( & 17) & 4.72750 & -.25350 & 1.86171 & ( & 2) \\
\hline C ( & 18) & 4.26069 & .92324 & 1.28288 & ( & 2) \\
\hline C ( & 19) & 1.00746 & -2.89150 & .83888 & ( & 2) \\
\hline C ( & $20)$ & -.14737 & -2.84842 & .03042 & ( & 2) \\
\hline Ci & 21) & -1.41445 & -2.63900 & .61315 & i & 1 \\
\hline $\mathrm{C}($ & 22) & -1.53845 & -2.68800 & 2.00748 & ( & ) \\
\hline C ( & 23) & -.41517 & -2.84209 & 2.81348 & ( & 2) \\
\hline C ( & $24)$ & .84764 & -2.91558 & 2.23389 & ( & 2) \\
\hline 01 & $25)$ & .14811 & 2.40836 & -1.60967 & ( & 6) \\
\hline C ( & 26) & -2.40196 & 2.70777 & -.29740 & ( & 1) \\
\hline O( & 27) & -1.75003 & .13050 & -1.31685 & ( & 6) \\
\hline $\mathrm{C}($ & $28)$ & -2.64460 & -2.32351 & -.21581 & ( & 1) \\
\hline 0( & 29) & -.03012 & -2.76208 & -1.32555 & ( & 6) \\
\hline $\mathrm{C}($ & $30)$ & 2.44011 & -2.87026 & .33706 & ( & 1) \\
\hline O( & 31) & 1.72300 & -.38463 & -1.00417 & ( & 6) \\
\hline $\mathrm{C}($ & $32)$ & 2.65872 & 2.17859 & -.22810 & ( & 1) \\
\hline $\mathrm{H}($ & $33)$ & -4.48994 & 2.46224 & 1.49457 & ( & 5) \\
\hline $\mathrm{H}($ & $34)$ & -4.71065 & -1.82664 & 1.54659 & ( & 5) \\
\hline $\mathrm{H}($ & $35)$ & 2.29028 & 2.76392 & 2.40195 & ( & 5) \\
\hline $\mathrm{H}($ & $36)$ & -1.99286 & 3.19689 & 2.34185 & ( & 5) \\
\hline $\mathrm{H}($ & $37)$ & 4.48898 & -2.38583 & 2.03593 & ( & 5) \\
\hline $\mathrm{H}($ & $38)$ & 4.66756 & 1.88605 & 1.63392 & ( & 5) \\
\hline $\mathrm{H}($ & 39) & -2.52579 & -2.56885 & 2.48248 & ( & 5) \\
\hline $\mathrm{H}($ & $40)$ & 1.73187 & -2.96386 & 2.89226 & ( & 5) \\
\hline $\mathrm{H}($ & 41) & -2.25888 & 2.68508 & -1.40353 & ( & 5) \\
\hline $\mathrm{H}($ & $42)$ & -2.35988 & -2.31015 & -1.29288 & ( & 5) \\
\hline $\mathrm{H}($ & $43)$ & 2.97358 & -3.64591 & .94381 & ( & 5) \\
\hline $\mathrm{H}($ & $44)$ & 2.47067 & 2.05606 & -1.31855 & ( & 5) \\
\hline $\mathrm{C}($ & $45)$ & .34896 & 3.52973 & -2.45648 & ( & 1) \\
\hline $\mathrm{C}($ & 46) & -.21080 & -3.99706 & -2.00430 & ( & . \\
\hline
\end{tabular}




\begin{tabular}{|c|c|c|c|c|}
\hline 47) & -2.13425 & .12198 & -2.68442 & ( \\
\hline 48$)$ & 2.07527 & -.46048 & -2.37858 & ( \\
\hline 49) & .35403 & 3.19330 & -3.51324 & 1 \\
\hline 50) & -.47161 & 4.27465 & -2.32923 & ( \\
\hline 51) & 1.32574 & 4.02450 & -2.23951 & ( \\
\hline 52) & -.04481 & -3.84536 & -3.09035 & ( \\
\hline 53) & .51256 & -4.76510 & -1.64018 & ( \\
\hline 54) & -1.24795 & -4.38332 & -1.85861 & ( \\
\hline 55) & -2.68423 & 1.05588 & -2.95049 & ( \\
\hline 56) & -1.22852 & .05840 & -3.32047 & ( \\
\hline 57) & -2.78482 & -.75589 & -2.91181 & ( \\
\hline 58) & 1.15339 & -.46685 & -2.99398 & ( \\
\hline 59) & 2.69378 & .41486 & -2.68662 & ( \\
\hline 60) & 2.64726 & -1.39045 & -2.59936 & ( \\
\hline 61) & -5.53857 & .37736 & 2.31486 & ( \\
\hline 62) & .15747 & 3.23552 & 3.58340 & ( \\
\hline 63) & 5.50824 & -.21231 & 2.64032 & ( \\
\hline 64) & -.52166 & -2.85384 & 3.91159 & ( \\
\hline 65) & -3.56265 & -3.38669 & -.00132 & ( \\
\hline 66) & -3.20920 & 3.83151 & .02755 & ( \\
\hline 67) & 3.55674 & 3.26560 & -.04737 & ( \\
\hline 68) & 2.52942 & -3.26319 & -1.02128 & ( \\
\hline 69) & -2.76853 & 5.04310 & -.56282 & ( \\
\hline 70$)$ & 4.57677 & 3.31020 & -1.03337 & ( \\
\hline 71) & 3.83641 & -3.65822 & -1.40923 & ( \\
\hline 72) & -4.52711 & -3.50086 & -1.03625 & ( \\
\hline 73) & -1.73641 & 5.30231 & -.22732 & ( \\
\hline 74$)$ & -2.79309 & 4.97028 & -1.67644 & ( \\
\hline 75) & -3.44722 & 5.86460 & -.25572 & ( \\
\hline 76) & 5.27244 & 4.14193 & -.80080 & ( \\
\hline 77) & 5.16092 & 2.35966 & -1.05245 & ( \\
\hline 78) & 4.13883 & 3.49474 & -2.04388 & ( \\
\hline 79) & 4.17642 & -4.53935 & -.81399 & , \\
\hline 80$)$ & 3.82583 & -3.94797 & -2.47967 & ( \\
\hline 81) & 4.56344 & -2.82116 & -1.28225 & ( \\
\hline 82$)$ & -5.10667 & -2.55453 & -1.15318 & ( \\
\hline 83$)$ & -4.03536 & -3.75497 & -2.00602 & ( \\
\hline 84$)$ & -5.23707 & -4.31477 & -.78460 & ( \\
\hline
\end{tabular}


Rcct isomer of 15, partial cone conformation (diequatorial-isoclinal (I)-isoclinal(II))

This structure has 0 imaginary frequencies, final steric energy is $37.8347 \mathrm{Kcal} / \mathrm{mol}$.

\begin{tabular}{|c|c|c|c|c|c|c|}
\hline & TES & $\triangle A T E D$ TC & DRIG & & & \\
\hline C ( & 1) & 2.85618 & -.24969 & .13171 & ( & 2) \\
\hline C ( & 2) & 2.72701 & -1.40015 & -.67227 & ( & \\
\hline Ci & 3) & 2.84277 & -1.26960 & -2.06449 & ( & \\
\hline C ( & 4) & 2.99955 & -.01761 & -2.65065 & ( & \\
\hline $\mathrm{C}($ & 5) & 3.00022 & 1.12571 & -1.85632 & ( & \\
\hline C ( & 6) & 2.89626 & 1.02992 & -.46168 & ( & \\
\hline C ( & 7) & 2.76407 & 2.27711 & .39326 & ( & \\
\hline C ( & 8) & .25686 & 2.73031 & .06853 & ( & \\
\hline C ( & 9) & 1.37069 & 2.56996 & .91508 & ( & \\
\hline C ( & 10) & 1.18134 & 2.64986 & 2.30307 & ( & \\
\hline Ci & 11) & -.09332 & 2.81086 & 2.83998 & ( & \\
\hline C ( & 12) & -1.20159 & 2.84218 & 1.99823 & ( & \\
\hline C ( & 13) & -1.04393 & 2.75541 & .60908 & ( & \\
\hline $\mathrm{C}($ & 14) & -2.27860 & 2.63091 & -.26097 & ( & \\
\hline $\mathrm{C}($ & 15) & -2.69851 & .14040 & -.47547 & ( & \\
\hline C ( & 16) & -3.09615 & 1.38911 & .03996 & ( & \\
\hline C ( & 17) & -4.19233 & 1.44323 & .91204 & ( & \\
\hline $\mathrm{C}($ & 18) & -4.83425 & .27574 & 1.31809 & ( & \\
\hline C ( & 19) & -4.35764 & -.96322 & .89750 & ( & \\
\hline $\mathrm{C}($ & $20)$ & -3.26756 & -1.04857 & .01982 & ( & \\
\hline C ( & 21) & -2.63763 & -2.38609 & -.31835 & ( & \\
\hline $\mathrm{C}($ & 22) & -.12951 & -2.84875 & -.16344 & ( & \\
\hline C ( & 23) & -1.37548 & -2.67356 & .46919 & ( & \\
\hline C ( & 24) & -1.43747 & -2.71637 & 1.86673 & ( & \\
\hline C ( & 25) & -.27380 & -2.84316 & 2.62352 & ( & \\
\hline C ( & 26) & .96639 & -2.88720 & 1.99199 & ( & \\
\hline C ( & 27) & 1.05742 & -2.85437 & .59264 & ( & \\
\hline Ci & 28) & 2.39896 & -2.77085 & -.10916 & ( & \\
\hline C ( & 29) & 3.82405 & -.42702 & 2.27436 & ( & \\
\hline C ( & $30)$ & 4.60425 & 3.60070 & -.29889 & ( & \\
\hline Ci & $31)$ & .46663 & 3.81953 & -1.99270 & ( & \\
\hline C ( & 32) & -3.92501 & 4.09488 & -1.12654 & ( & \\
\hline C ( & 33) & -1.75218 & .10017 & -2.63075 & ( & \\
\hline C ( & $34)$ & -4.48591 & -3.65366 & -1.08188 & ( & \\
\hline C ( & $35)$ & -.18877 & -3.98155 & -2.20956 & ( & \\
\hline Ci & $36)$ & 4.64549 & -3.49634 & .12112 & ( & \\
\hline 01 & 37) & 2.65093 & -.34060 & 1.47787 & ( & \\
\hline O( & $38)$ & 3.19480 & 3.43356 & -.31120 & ( & \\
\hline 0( & 39) & .41407 & 2.59250 & -1.27922 & ( & \\
\hline O( & $40)$ & -3.04767 & 3.80693 & -.04873 & ( & 7 \\
\hline O( & 41) & -1.56023 & .06646 & -1.22451 & ( & 6) \\
\hline 0( & 42) & -3.53418 & -3.45523 & -.04775 & ( & \\
\hline 0( & 431 & -.04667 & -2.74710 & -1.52204 & ( & \\
\hline O( & $44)$ & 3.42469 & -3.19085 & .77703 & ( & \\
\hline $\mathrm{H}($ & $45)$ & 2.76940 & -2.15696 & -2.71588 & ( & \\
\hline $\mathrm{H}($ & 46) & 3.04549 & 2.11177 & -2.34683 & ( & \\
\hline $\mathrm{H}($ & 47) & 3.42861 & 2.14747 & 1.28014 & ( & \\
\hline
\end{tabular}




\begin{tabular}{|c|c|c|c|c|}
\hline 48) & 2.04009 & 2.55872 & 2.98958 & ( \\
\hline 49) & -2.21065 & 2.89851 & 2.43893 & 1 \\
\hline 50$)$ & -1.96735 & 2.59683 & -1.33004 & 1 \\
\hline 51) & -4.53053 & 2.40796 & 1.32522 & ( \\
\hline 52) & -4.82428 & -1.87727 & 1.30052 & ( \\
\hline 53) & -2.39489 & -2.39080 & -1.40585 & 1 \\
\hline 54) & -2.40718 & -2.61069 & 2.38098 & ( \\
\hline 55) & 1.87833 & -2.91662 & 2.61045 & ( \\
\hline 56) & 4.41606 & -1.33725 & 2.02502 & ( \\
\hline 57) & 3.53594 & -.48217 & 3.34388 & 1 \\
\hline 58) & 4.47506 & .46739 & 2.13340 & ( \\
\hline 59) & 4.96837 & 3.78764 & .74012 & ( \\
\hline 60$)$ & 4.87253 & 4.47771 & -.92241 & ( \\
\hline 61) & 5.12036 & 2.70327 & -.71529 & ( \\
\hline 62) & -.48212 & 4.39437 & -1.86916 & ( \\
\hline 63$)$ & .60797 & 3.60888 & -3.07233 & ( \\
\hline 64$)$ & 1.31702 & 4.45109 & -1.64357 & ( \\
\hline 65) & -3.34665 & 4.31900 & -2.05510 & ( \\
\hline 66) & -4.53265 & 4.98740 & -.87352 & ( \\
\hline 67) & -4.61836 & 3.24231 & -1.32036 & ( \\
\hline 68$)$ & -2.37419 & -.76189 & -2.97001 & ( \\
\hline 69) & -.76627 & .04005 & -3.13543 & ( \\
\hline 70$)$ & -2.25048 & 1.04813 & -2.94411 & ( \\
\hline 71) & -5.07335 & -2.72436 & -1.27281 & ( \\
\hline 72$)$ & -5.19018 & -4.45548 & -.78031 & ( \\
\hline 73) & -3.98022 & -3.97108 & -2.02554 & ( \\
\hline 74$)$ & .60906 & -4.70094 & -1.90659 & ( \\
\hline 75$)$ & -.10351 & -3.80403 & -3.30097 & ( \\
\hline 76) & -1.18599 & -4.43948 & -2.00459 & ( \\
\hline 77) & 3.06590 & .07410 & -3.74819 & ( \\
\hline 78$)$ & -.23041 & 2.86034 & 3.93364 & ( \\
\hline 79) & -5.68104 & .32966 & 2.02341 & ( \\
\hline 80$)$ & -.33199 & -2.85438 & 3.72523 & ( \\
\hline 81) & 2.35707 & -3.50571 & -.95064 & ( \\
\hline 82$)$ & 5.38929 & -3.82875 & .87353 & ( \\
\hline 83) & 4.50298 & -4.32241 & -.61614 & ( \\
\hline 84$)$ & 5.05636 & -2.59942 & -.40049 & ( \\
\hline
\end{tabular}




\section{Rctt isomer of $\mathbf{1 5}$, cone conformation (diequatorial-diaxial)}

This structure has $42.3357 \mathrm{Kcal} / \mathrm{mol}$.
0 imaginary frequencies. Final steric energy is

COORDINATES TRANSLATED TO NEW ORIGIN WHICH IS CENTER OF MASS

\begin{tabular}{|c|c|c|c|c|}
\hline C ( & 3.11851 & -1.41365 & -.05251 & ( \\
\hline C ( & 2.78402 & -.16152 & .49685 & 1 \\
\hline C ( & 3.30636 & 1.02067 & -.06046 & ( \\
\hline C ( & 4.33971 & .92043 & -1.00384 & ( \\
\hline C ( & 4.78227 & -.32473 & -1.44341 & ( \\
\hline C ( & 4.15413 & -1.48354 & -.99483 & ( \\
\hline C ( & 1.41376 & 2.63923 & -.49818 & ( \\
\hline C ( & .14492 & 2.72336 & .11466 & ( \\
\hline 9) & -1.02526 & 2.76019 & -.67502 & ( \\
\hline 10) & -.89015 & 2.87785 & -2.06785 & ( \\
\hline 11) & .36387 & 2.90089 & -2.66978 & ( \\
\hline 12) & 1.50775 & 2.76637 & -1.89046 & ( \\
\hline 13) & -3.18808 & 1.39373 & -.51290 & ( \\
\hline 14) & -2.79415 & .12270 & -.04511 & ( \\
\hline 15) & -3.36245 & -1.04320 & -.60125 & ( \\
\hline 16) & -4.45895 & -.91595 & -1.46721 & ( \\
\hline 17) & -4.93351 & .33940 & -1.83484 & ( \\
\hline 18) & -4.28727 & 1.48493 & -1.38071 & ( \\
\hline 19) & -1.38694 & -2.64203 & -.83826 & ( \\
\hline 20) & -.25199 & -2.79546 & -.01504 & ( \\
\hline 21) & 1.04112 & -2.75617 & -.57489 & ( \\
\hline 22) & 1.17956 & -2.78352 & -1.96864 & ( \\
\hline 23) & .05930 & -2.74622 & -2.79271 & ( \\
\hline 24) & -1.21009 & -2.64864 & -2.23109 & ( \\
\hline 25) & .04915 & 2.52393 & 1.46071 & ( \\
\hline 26) & 2.68967 & 2.36628 & .27754 & ( \\
\hline 27) & 1.73532 & -.07728 & 1.36425 & ( \\
\hline 28) & 2.28849 & -2.63913 & .28022 & ( \\
\hline 29) & -.37549 & -2.73515 & 1.34157 & ( \\
\hline 30) & -2.81061 & -2.43653 & -.35381 & ( \\
\hline 31) & -1.67625 & .01331 & .72692 & ( \\
\hline 32) & -2.45275 & 2.67564 & -.16287 & ( \\
\hline 33) & 4.78955 & 1.82700 & -1.44111 & ( \\
\hline 34$)$ & 4.45345 & -2.45339 & -1.42582 & ( \\
\hline 35) & -1.78745 & 2.92453 & -2.70852 & ( \\
\hline 36) & 2.49221 & 2.72529 & -2.38541 & ( \\
\hline 37) & -4.94236 & -1.81227 & -1.89178 & ( \\
\hline 38) & -4.63782 & 2.46860 & -1.73690 & ( \\
\hline 39) & 2.18136 & -2.79653 & -2.42781 & ( \\
\hline 40$)$ & -2.08188 & -2.54737 & -2.90004 & ( \\
\hline 41) & 2.49695 & 2.39095 & 1.37558 & ( \\
\hline 42) & 1.99056 & -2.58584 & 1.35278 & ( \\
\hline 43$)$ & -3.42269 & -3.16481 & -.94543 & ( \\
\hline 44$)$ & -2.98293 & 3.52495 & -.66596 & ( \\
\hline 45$)$ & .09350 & 3.70298 & 2.25177 & ( \\
\hline 46) & -.39101 & -4.00414 & 1.97998 & ( \\
\hline
\end{tabular}




\begin{tabular}{|c|c|c|c|c|}
\hline 47) & 2.12082 & -.10203 & 2.73186 & 1) \\
\hline 48$)$ & -1.90464 & -.03466 & 2.12847 & 1) \\
\hline 49$)$ & -.15882 & 3.44864 & 3.30157 & 5) \\
\hline 50) & 1.11466 & 4.14946 & 2.23938 & 5) \\
\hline 51) & -.63462 & 4.46527 & 1.88670 & 5) \\
\hline 52) & -.54499 & -3.86394 & 3.06933 & 5) \\
\hline 53) & -1.21798 & -4.64156 & 1.58532 & ( \\
\hline 54$)$ & .57791 & -4.53743 & 1.82834 & 5) \\
\hline 55) & 2.80832 & .74475 & 2.96846 & ( \\
\hline 56) & 1.22184 & -.00719 & 3.37281 & 5) \\
\hline 57) & 2.63144 & -1.06201 & 2.98448 & 5) \\
\hline 58) & -.94333 & -.19802 & 2.65311 & 5) \\
\hline 59) & -2.34291 & .91789 & 2.50222 & 5) \\
\hline 60$)$ & -2.59344 & -.86515 & 2.40236 & 5) \\
\hline 61) & 5.58403 & -.38939 & -2.19869 & 5) \\
\hline 62) & .44976 & 2.97884 & -3.76697 & 5) \\
\hline 63) & -5.78972 & .42545 & -2.52552 & 5) \\
\hline 64$)$ & .18112 & -2.74153 & -3.88927 & 5) \\
\hline 65) & 3.64424 & 3.37098 & -.03985 & 6) \\
\hline 66) & 3.03858 & -3.82762 & .07180 & 6) \\
\hline 67) & -2.95576 & -2.77807 & 1.01356 & 6) \\
\hline 68) & -2.52941 & 2.90690 & 1.23245 & 6) \\
\hline 69) & 3.34517 & 4.64781 & .49784 & 1) \\
\hline 70) & 3.95485 & -4.09247 & 1.12278 & 1) \\
\hline 71) & -4.30401 & -2.95566 & 1.41939 & 1) \\
\hline 72) & -3.83666 & 3.21514 & 1.69136 & 1) \\
\hline 73) & 2.35867 & 5.01830 & .13121 & 5) \\
\hline 74) & 3.34431 & 4.61355 & 1.61346 & 5) \\
\hline 75) & 4.12468 & 5.36822 & .17666 & 5) \\
\hline 76) & 3.41190 & -4.27626 & 2.08106 & 5) \\
\hline 77) & 4.53927 & -5.00212 & .87623 & 5) \\
\hline 78) & 4.66748 & -3.24471 & 1.25917 & 5) \\
\hline 79) & -4.79182 & -3.76793 & .82919 & 5) \\
\hline 80$)$ & -4.32556 & -3.24525 & 2.48970 & 5) \\
\hline 81) & -4.88612 & -2.01061 & 1.30436 & 5) \\
\hline 82) & -4.53733 & 2.36583 & 1.50978 & 5) \\
\hline 83$)$ & -3.80229 & 3.40566 & 2.78334 & 5) \\
\hline 84$)$ & -4.22644 & 4.13332 & 1.19027 & 5) \\
\hline
\end{tabular}


Rctt isomer of 15, partial cone conformation (diequatorial-diisoclinal (II))

This structure has

0 imaginary frequencies. Final steric energy is $37.3780 \mathrm{Kcal} / \mathrm{mol}$.

\begin{tabular}{|c|c|c|c|c|c|c|}
\hline & & ED & & & & \\
\hline C ( & 1) & .03483 & -2.89002 & .32286 & ( & 2) \\
\hline $\mathrm{C}($ & 2) & -1.18494 & -2.83639 & -.38012 & ( & 2) \\
\hline C ( & 3) & -1.16196 & -2.94023 & -1.77812 & ( & 2) \\
\hline C ( & 4) & .04563 & -3.03334 & -2.46455 & ( & 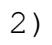 \\
\hline C ( & 5) & 1.24943 & -2.97367 & -1.76734 & ( & \\
\hline C ( & 6) & 1.26134 & -2.85741 & -.37052 & ( & \\
\hline C ( & 7) & 2.56007 & -2.63650 & .38220 & ( & \\
\hline $\mathrm{C}($ & $8)$ & 2.80436 & -.11316 & -.00191 & ( & \\
\hline C ( & 9) & 2.76350 & -1.21585 & .87311 & ( & \\
\hline C ( & 10) & 2.86146 & -.98890 & 2.25384 & ( & \\
\hline $\mathrm{C}($ & 11) & 2.92971 & .30755 & 2.75800 & ( & \\
\hline C ( & 12) & 2.84495 & 1.39445 & 1.89185 & ( & ) \\
\hline C ( & 13) & 2.73339 & 1.19701 & .50960 & ( & ) \\
\hline C ( & 14) & 2.48416 & 2.39328 & -.38644 & ( & \\
\hline $\mathrm{C}($ & 15) & -.03756 & 2.62703 & -.54629 & ( & , \\
\hline $\mathrm{C}($ & 16) & 1.19300 & 3.12601 & -.07544 & ( & ) \\
\hline C ( & 17) & 1.19364 & 4.25891 & .75011 & ( & ) \\
\hline $\mathrm{C}($ & 18) & -.00385 & 4.85026 & 1.14271 & ( & 2) \\
\hline $\mathrm{C}($ & 19) & -1.21786 & 4.28647 & .75866 & ( & , \\
\hline $\mathrm{C}($ & $20)$ & -1.25288 & 3.14769 & -.05978 & ( & ) \\
\hline $\mathrm{C}($ & 21) & -2.56704 & 2.44524 & -.35159 & ( & ) \\
\hline C ( & 22) & -2.78394 & -.09188 & -.02069 & ( & ) \\
\hline $\mathrm{C}($ & $23)$ & -2.79419 & 1.21605 & .50714 & ( & 1 \\
\hline C ( & 24) & -2.96772 & 1.39239 & 1.88586 & ( & ) ) \\
\hline $\mathrm{C}($ & $25)$ & -3.05662 & .29392 & 2.73622 & ( & ) \\
\hline C ( & 26) & -2.92547 & -.99267 & 2.22216 & ( & ) \\
\hline $\mathrm{C}($ & 27) & -2.74682 & -1.20235 & .84572 & ( & 2) \\
\hline C ( & 28) & -2.48743 & -2.61826 & .36669 & ( & L) \\
\hline C ( & 29) & .02276 & -3.88759 & 2.44972 & ( & ) \\
\hline C ( & $30)$ & 4.87099 & -3.16874 & .27072 & ( & ) \\
\hline C ( & 31) & 3.83398 & -.26916 & -2.10310 & ( & L) \\
\hline C ( & $32)$ & 3.79675 & 4.11501 & -1.34451 & ( & L) \\
\hline C ( & $33)$ & -.05423 & 1.59297 & -2.66151 & ( & ) \\
\hline C ( & $34)$ & -4.87332 & 2.97275 & -.64191 & ( & L) \\
\hline $\mathrm{C}($ & $35)$ & -3.68014 & -.34574 & -2.18214 & ( & 1 \\
\hline C ( & $36)$ & -3.83284 & -4.40460 & -.41944 & ( & ) \\
\hline 0( & $37)$ & .02929 & -2.69713 & 1.67448 & ( & 6) \\
\hline O( & $38)$ & 3.65196 & -3.02019 & -.44017 & ( & 6) \\
\hline 01 & $39)$ & 2.63644 & -.31315 & -1.34073 & ( & 6) \\
\hline 01 & $40)$ & 3.60488 & 3.25376 & -.23276 & ( & (1) \\
\hline 01 & 41) & -.04872 & 1.45724 & -1.24819 & ( & 6) \\
\hline 01 & $42)$ & -3.62022 & 3.36979 & -.11129 & ( & 6) \\
\hline 01 & $43)$ & -2.53272 & -.27526 & -1.34876 & ( & 6) \\
\hline O( & $44)$ & -3.60449 & -3.00374 & -.42186 & ( & \\
\hline $\mathrm{H}($ & $45)$ & -2.10050 & -2.91826 & -2.35556 & ( & 5) \\
\hline $\mathrm{H}($ & 46) & 2.19355 & -2.97863 & -2.33617 & ( & 5) \\
\hline $\mathrm{H}($ & 47) & 2.53837 & -3.32427 & 1.26246 & ( & \\
\hline
\end{tabular}




\begin{tabular}{|c|c|c|c|c|}
\hline 48) & 2.85468 & -1.83642 & 2.96006 & 5) \\
\hline 49) & 2.82661 & 2.41521 & 2.30821 & 5) \\
\hline 50$)$ & 2.44306 & 2.04992 & -1.44522 & 5) \\
\hline 51) & 2.14207 & 4.67495 & 1.12851 & 5) \\
\hline 52) & -2.15132 & 4.73065 & 1.14168 & 5) \\
\hline 53) & -2.57338 & 2.17501 & -1.43327 & 5) \\
\hline 54) & -3.00087 & 2.40833 & 2.31360 & 5) \\
\hline 55) & -2.93334 & -1.84677 & 2.92041 & 5) \\
\hline 56) & -.88079 & -4.50446 & 2.23072 & ( \\
\hline 57) & .01139 & -3.62226 & 3.52649 & 5) \\
\hline 58$)$ & .93236 & -4.50183 & 2.24949 & 5) \\
\hline 59) & 4.32766 & .72791 & -2.01573 & 5) \\
\hline 60$)$ & 3.59398 & -.44662 & -3.17120 & 5) \\
\hline 61) & 4.55182 & -1.05439 & -1.76959 & 5) \\
\hline 62) & 4.03905 & 3.52544 & -2.26136 & 5) \\
\hline 63) & 4.64735 & 4.79534 & -1.13601 & 5) \\
\hline 64$)$ & 2.88956 & 4.73630 & -1.53452 & 5) \\
\hline 65) & -.96826 & 2.13101 & -3.00792 & 5) \\
\hline 66) & -.04503 & .58545 & -3.12536 & 5) \\
\hline 67$)$ & .84567 & 2.15029 & -3.01436 & 5) \\
\hline 68$)$ & -4.82185 & 2.89410 & -1.75413 & 5) \\
\hline 69) & -5.63433 & 3.73774 & -.38610 & 5) \\
\hline 70$)$ & -5.20309 & 1.99806 & -.21049 & 5) \\
\hline 71) & -4.38202 & -1.14106 & -1.83969 & 5) \\
\hline 72) & -3.36474 & -.57954 & -3.21937 & 5) \\
\hline 73) & -4.22228 & .62794 & -2.19596 & 5) \\
\hline 74$)$ & .04993 & -3.10137 & -3.56579 & 5) \\
\hline 75$)$ & 2.99438 & .47485 & 3.84665 & 5) \\
\hline 76) & .00849 & 5.73428 & 1.80289 & 5) \\
\hline 77) & -3.17879 & .44601 & 3.82215 & 5) \\
\hline 78) & -2.46439 & -3.27258 & 1.27098 & 5) \\
\hline 79) & 5.66478 & -3.49238 & -.43285 & 5) \\
\hline 80$)$ & 5.18832 & -2.20276 & .73051 & 5) \\
\hline 81$)$ & 4.77322 & -3.94529 & 1.06672 & 5) \\
\hline 82) & -4.11038 & -4.75547 & .60374 & 5) \\
\hline 83$)$ & -4.67303 & -4.63781 & -1.10471 & 5) \\
\hline 84$)$ & -2.93096 & -4.96111 & -.76866 & ( \\
\hline
\end{tabular}


Rctt isomer of 15, partial cone conformation (diequatorial-diisoclinal (II))

This structure has

0 imaginary frequencies, final steric energy is $37.9770 \mathrm{KCAL}$.

\begin{tabular}{|c|c|c|c|c|c|c|}
\hline ז & TES & TRANSLATED TO & NEW ORIGIN & IS & & \\
\hline $\mathrm{C}($ & 1) & 2.92630 & -.00086 & .31865 & ( & 2) \\
\hline C ( & 2) & 2.82964 & -1.22313 & -.37477 & ( & 2) \\
\hline $\mathrm{C}($ & 3) & 2.84245 & -1.20585 & -1.77671 & ( & 21 \\
\hline C ( & 4) & 2.87452 & -.00037 & -2.47207 & ( & 2) \\
\hline C ( & 5) & 2.84299 & 1.20528 & -1.77608 & ( & 21 \\
\hline C ( & 6) & 2.83032 & 1.22211 & -.37460 & ( & 21 \\
\hline $\mathrm{C}($ & 7) & 2.63091 & 2.51836 & .38869 & ( & 1 \\
\hline $\mathrm{C}($ & 8) & .10460 & 2.82133 & .03934 & ( & 2) \\
\hline C ( & 9) & 1.21851 & 2.73641 & .89748 & ( & 1 \\
\hline C ( & 10) & 1.00824 & 2.79389 & 2.28333 & ( & 2) \\
\hline Ci & 11) & -.28127 & 2.85665 & 2.80631 & ( & 2) \\
\hline $\mathrm{C}($ & 12) & -1.37946 & 2.80611 & 1.95242 & ( & 2) \\
\hline C ( & 13) & -1.19958 & 2.73936 & .56485 & ( & ) \\
\hline $\mathrm{C}($ & 14) & -2.40888 & 2.51940 & -.32164 & ( & 1) \\
\hline $\mathrm{C}($ & 15) & -2.63153 & .00073 & -.50839 & ( & 2) \\
\hline $\mathrm{C}($ & 16) & -3.14115 & 1.22320 & -.02964 & ( & 2) \\
\hline C ( & 17) & -4.27668 & 1.20723 & .79291 & ( & 2) \\
\hline $\mathrm{C}($ & 18) & -4.85527 & .00149 & 1.18047 & ( & 2) \\
\hline C ( & 19) & -4.27710 & -1.20500 & .79309 & ( & 2) \\
\hline $\mathrm{C}($ & $20)$ & -3.14201 & -1.22175 & -.02931 & ( & 2) \\
\hline C ( & 21) & -2.41048 & -2.51838 & -.32121 & ( & 1) \\
\hline C ( & 22) & .10396 & -2.82135 & .03922 & ( & 21 \\
\hline $\mathrm{C}($ & 23) & -1.20138 & -2.73918 & .56537 & ( & 2) \\
\hline C ( & 24) & -1.38099 & -2.80608 & 1.95166 & ( & 2) \\
\hline C ( & $25)$ & -.28176 & -2.85773 & 2.80620 & ( & 2) \\
\hline $\mathrm{C}($ & 26) & 1.00658 & -2.79558 & 2.28361 & ( & 2) \\
\hline Ci & 27) & 1.21693 & -2.73719 & .89650 & ( & 2) \\
\hline $\mathrm{C}($ & 28) & 2.62949 & -2.51957 & .38799 & ( & 1) \\
\hline C ( & 29) & 4.06471 & -.00140 & 2.36991 & ( & 1) \\
\hline Ci & $30)$ & 4.38042 & 3.87030 & -.46328 & ( & 1) \\
\hline C ( & 31) & .24651 & 3.93609 & -2.01337 & ( & 1) \\
\hline C ( & 32) & -4.12831 & 3.87069 & -1.22847 & ( & 1) \\
\hline $\mathrm{C}($ & 33) & -1.57718 & .00019 & -2.61492 & ( & 1) \\
\hline $\mathrm{C}($ & $34)$ & -4.13068 & -3.86843 & -1.22843 & ( & 1) \\
\hline C ( & $35)$ & .24463 & -3.93536 & -2.01399 & ( & 1) \\
\hline $\mathrm{C}($ & $36)$ & 4.37864 & -3.87185 & -.46417 & ( & 1) \\
\hline O( & $37)$ & 2.82304 & -.00103 & 1.67984 & ( & 6) \\
\hline 01 & 38) & 2.98055 & 3.64412 & -.40338 & ( & 6) \\
\hline O( & 39) & .28436 & 2.70405 & -1.30744 & ( & 6) \\
\hline 0( & $40)$ & -3.26249 & 3.63914 & -.12791 & ( & 6) \\
\hline O( & 41) & -1.45539 & .00032 & -1.20051 & ( & 6) \\
\hline 0( & 42) & -3.26480 & -3.63762 & -.12776 & ( & 6) \\
\hline 0( & $43)$ & .28345 & -2.70363 & -1.30755 & ( & 6) \\
\hline O( & 44) & 2.97884 & -3.64518 & -.40443 & ( & 6) \\
\hline $\mathrm{H}($ & $45)$ & 2.78310 & -2.14709 & -2.34728 & ( & 5) \\
\hline
\end{tabular}




\begin{tabular}{|c|c|c|c|c|}
\hline 46) & 2.78380 & 2.14670 & -2.34638 & ( \\
\hline 47) & 3.30664 & 2.49849 & 1.27727 & ( \\
\hline 48$)$ & 1.86332 & 2.75665 & 2.97938 & ( \\
\hline 49) & -2.39506 & 2.77937 & 2.38093 & ( \\
\hline 50) & -2.08032 & 2.50171 & -1.38592 & ( \\
\hline 51) & -4.70040 & 2.14903 & 1.17917 & ( \\
\hline 52) & -4.70118 & -2.14647 & 1.17978 & ( \\
\hline 53) & -2.08168 & -2.50082 & -1.38541 & ( \\
\hline 54) & -2.39645 & -2.77811 & 2.38041 & ( \\
\hline 55) & 1.86187 & -2.75999 & 2.97950 & ( \\
\hline 56) & 4.66330 & -.90855 & 2.11675 & ( \\
\hline 57) & 3.87565 & -.00152 & 3.46271 & ( \\
\hline 58) & 4.66370 & .90555 & 2.11702 & ( \\
\hline 59) & -.74961 & 4.42780 & -1.90480 & ( \\
\hline 60$)$ & .42499 & 3.74560 & -3.09125 & ( \\
\hline 61) & 1.03341 & 4.63526 & -1.64469 & ( \\
\hline 62) & -3.54218 & 4.14114 & -2.13964 & ( \\
\hline 63) & -4.80461 & 4.71625 & -.98866 & ( \\
\hline 64) & -4.75359 & 2.97226 & -1.44526 & ( \\
\hline 65) & -2.12179 & -.90679 & -2.96960 & ( \\
\hline 66) & -.56504 & -.00014 & -3.06863 & ( \\
\hline 67) & -2.12129 & .90738 & -2.96982 & ( \\
\hline 68$)$ & -4.75542 & -2.96959 & -1.44508 & ( \\
\hline 69) & -4.80749 & -4.71364 & -.98886 & ( \\
\hline 70$)$ & -3.54464 & -4.13903 & -2.13962 & ( \\
\hline 71) & 1.03124 & -4.63515 & -1.64588 & ( \\
\hline 72$)$ & .42284 & -3.74452 & -3.09185 & ( \\
\hline 73) & -.75174 & -4.42653 & -1.90527 & ( \\
\hline 74$)$ & 2.86146 & -.00017 & -3.57531 & ( \\
\hline 75$)$ & -.43395 & 2.88777 & 3.89858 & ( \\
\hline 76) & -5.73660 & .00178 & 1.84432 & ( \\
\hline 77$)$ & -.43443 & -2.88925 & 3.89847 & ( \\
\hline 78$)$ & 3.30501 & -2.50035 & 1.27675 & ( \\
\hline 79) & 4.58303 & 4.72097 & -1.14533 & ( \\
\hline 80$)$ & 4.77986 & 4.13061 & .54650 & 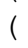 \\
\hline 81) & 4.91758 & 2.97321 & -.85282 & ( \\
\hline 82$)$ & 4.77782 & -4.13267 & .54558 & ( \\
\hline 83$)$ & 4.58106 & -4.72236 & -1.14648 & ( \\
\hline 84$)$ & 4.91620 & -2.97482 & -.85330 & ( \\
\hline
\end{tabular}

